\begin{tabular}{|c|l|}
\hline Title & $\begin{array}{l}\text { Use of gas chromatography-mass spectrometry- olfactometry and a conventional flask test to identify off-flavor } \\
\text { compounds generated from pheny lal anine during chlorination of drinking water }\end{array}$ \\
\hline Author(s) & Matsushita, Taku; Sakuma, Miki; Tazawa, Shiori; Hatase, Taiki; Shirasaki, Nobutaka; Matsui, Y oshihiko \\
\hline Citation & $\begin{array}{l}\text { Water research, 125, 332-340 } \\
\text { https://doi.org/10.1016/.watres.2017.08.063 }\end{array}$ \\
\hline Issue Date & 2017-11-15 \\
\hline Doc URL & http://hdl.handle.net/2115/76129 \\
\hline Rights & $\begin{array}{l}\text { O 2017. This manuscript version is made avail lable under the CC-BY-NC-ND 4.0 license } \\
\text { http://creativecommons.org/icenses/by-nc-nd/4.0/ }\end{array}$ \\
\hline Rights(URL) & http://creativecommons.org/icenses/by-nc-nd/4.0/ \\
\hline Type & article (author version) \\
\hline File Information & matsushita.pdf \\
\hline
\end{tabular}

Instructions for use 


\title{
Use of gas chromatography-mass spectrometry-olfactometry and a conventional flask test to identify off-flavor compounds generated from phenylalanine during chlorination of drinking water
}

\author{
Taku Matsushita*, Miki Sakuma, Shiori Tazawa, Taiki Hatase, \\ Nobutaka Shirasaki, and Yoshihiko Matsui \\ Graduate School of Engineering, Hokkaido University, N13W8, Sapporo 060-8628, Japan \\ *Corresponding author: e-mail, taku-m@eng.hokudai.ac.jp; Tel/Fax, +81-11-706-7279
}

\begin{abstract}
Off-flavor in drinking water can be caused by transformation products (TPs) generated from organic compounds, such as amino acids, present during chlorination. However, the contributions of many of these TPs to overall off-flavor have not been quantified, mainly because the lack of appropriate chemical standards prevents sensory evaluation by means of a conventional flask test. In the present study, we used gas chromatography-mass spectrometry-olfactometry (GC-MS-O) to identify compounds responsible for the off-flavor generated by chlorination of an aqueous solution of the amino acid phenylalanine, and we propose a sensory evaluation procedure for quantification of the contributions of the identified TPs to the overall off-flavor, regardless of the availability of chemical standards of the TPs. GC-MS-O revealed that two TPs, $N$-chlorophenylacetaldimine and 2-chloro-2phenylacetaldehyde, for which chemical standards are not commercially available, were the main components responsible for the off-flavor of the chlorinated solution. By using a sensory evaluation procedure involving a combination of GC-MS-O and a conventional flask test, we quantified the contributions of TPs to the overall off-flavor of the chlorinated solution. Approximately $60 \%$ of the off-flavor was attributable to free chlorine (13\%), 2-chloro-2-phenylacetaldehyde (13\%), trichloramine $(12 \%)$ phenylacetaldehyde $(11 \%)$ phenylacetonitrile $(8 \%)$, and $N$ chlorophenylacetaldimine (2\%). Treatment with powdered activated carbon (PAC) removed the offflavor. Experiments with chlorination of ${ }^{15} \mathrm{~N}$-labeled phenylalanine suggested that PAC reductively decomposed trichloramine into $\mathrm{N}_{2}$ gas and adsorbed all of the other identified TPs. Superfine PAC (median diameter, $0.7 \mu \mathrm{m}$ ) removed the off-flavor more rapidly than normal-size PAC (median diameter, $8.0 \mu \mathrm{m})$.
\end{abstract}

Keywords: Chlorination; chlorinous odor; disinfection by-product; powdered activated carbon; sensory evaluation.

\section{Introduction}

Off-flavors are an important issue in the field of drinking water treatment, particularly in treatment plants where free chlorine is used for disinfection. The primary contributor to chlorination-derived off-flavors is widely accepted to be trichloramine, which is formed mainly by reaction between ammonium nitrogen and free chlorine. In addition, organic nitrogen compounds, such as amino acids, can be transformed to off-flavor compounds during chlorination (Hrudey et al. 1988, Froese et al. 1999, Kajino et al. 1999). Off-flavor compounds formed by reactions between amino acids and free chlorine reportedly include aldehydes and $N$-chloroaldimines. For example, Hrudey et al. (1988) attributed off-flavor events involving Canadian drinking water to three amino-acid-derived aldehydes that were produced from amino acids during chlorination and were present at concentrations higher than their odor thresholds. Similarly, Freuze et al. (2005) suggested that $N$-chloroaldimines may have been the source of off-flavors in drinking water during an isolated odor episode in France. 
However, the contributions of individual compounds to overall off-flavor have not been quantified, because chlorination generates complex mixtures of various transformation products (TPs). Some TPs are commercially available, and accordingly their contributions, as well as their odor thresholds, can be directly evaluated by means of sensory evaluation tests involving chemical standards. However, most TPs are not commercially available, and therefore their contributions to overall off-flavor cannot be evaluated.

Gas chromatography-mass spectrometry-olfactometry (GC-MS-O) is a method involving the use of human assessors to detect and evaluate volatile compounds separated by GC and identified by MS (Delahunty et al. 2006). GC-MS-O is frequently used in the food, beverage, fragrance, and essential oil industries to elucidate the contributions of specific volatile compounds to flavor or fragrance and to identify the causes of off-flavors (Delahunty et al. 2006). In contrast, the use of GC-MS-O in the field of water treatment has been limited. Various investigators have attempted to use GC-MS-O to identify off-flavor compounds in lake water (Peter et al. 2009), ground water (Hochereau and Bruchet 2004), and tap water (Benanou et al. 2003, Hochereau and Bruchet 2004). Although these attempts succeeded to a certain extent - some of the off-flavor compounds were identified - the contributions of the identified compounds to overall off-flavor were not quantified.

In the present study, using an aqueous solution of the amino acid phenylalanine as a model system, we combined GC-MS-O and a conventional flask test to elucidate the contributions of TPs to the offflavor of a chlorinated phenylalanine-containing solution, without the need for chemical standards. Additionally, we investigated removal of the off-flavor by means of treatment with powdered activated carbon (PAC), both commercially available normal-size PAC (NPAC) and laboratoryprepared superfine PAC (SPAC). We conducted experiments with isotope-labeled phenylalanine and used mass balance to investigate the removal mechanism.

\section{Materials and Methods}

\subsection{Preparation of chlorinated solutions and their uses}

We prepared three chlorinated solutions, which are shown in Fig. 1, along with their uses. The first solution was prepared as follows. Phenylalanine (Wako Pure Chemicals Industries, Osaka, Japan) was dissolved in phosphate buffer $(0.1 \mathrm{mM}, \mathrm{pH} 7.0)$ at a final concentration of $1 \mu \mathrm{M}$. To this solution was added enough sodium hypochlorite to achieve a residual chlorine concentration of $1 \mathrm{mg}-\mathrm{Cl}_{2} / \mathrm{L}$ after $24 \mathrm{~h}$ of contact time. The solution was magnetically stirred for $30 \mathrm{~min}$ and then left at rest without headspace for $24 \mathrm{~h}$ at $20^{\circ} \mathrm{C}$ in the dark. The resulting solution (hereafter referred to as the chlorinated phenylalanine-containing solution) was used for elucidating the contributions of chlorination-derived TPs to its overall off-flavor. Specifically, the solution was subjected to a conventional flask test to evaluate the intensity of the off-flavor. In addition, the concentrations of phenylalanine was determined by liquid chromatography-tandem mass spectrometry (see SI). Monochloramine, dichloramine, and free chlorine were determined with the $N, N$-diethyl- $p$ phenylenediamine colorimetric method (American Public Health Association/American Water Works Association/Water Environment Federation 2005). TPs generated from phenylalanine during chlorination were investigated by means of purge-and-trap gas chromatography-mass spectrometry (P\&T-GC/MS); TPs for which chemical standards were commercially available were identified and quantified. Finally, a portion of the solution was subjected to liquid-liquid extraction (LLE), and another portion was subjected to solid-phase microextraction (SPME); each of the extracts was then analyzed by GC-MS-O to identify TPs that contributed to the off-flavor.

The second chlorinated solution was used to assess the off-flavor contribution of $N$ chlorophenylacetaldimine (NCPAAI), which is reported to be produced during the chlorination of phenylalanine-containing solutions (Conyers and Scully 1993, Freuze et al. 2004, Freuze et al. 2005) 
but is not commercially available. This second chlorinated solution (hereafter referred to as the highNCPAAI solution) was prepared as described by Freuze et al. (2004). Briefly, phosphate buffer (100 $\mathrm{mM}, \mathrm{pH}$ 7) was cooled in an ice bath, and sodium hypochlorite was added at a concentration of 17.0 $\mathrm{mg}-\mathrm{Cl}_{2} / \mathrm{L}$. Then phenylalanine $(100 \mu \mathrm{M})$ was added, and the resulting solution was stirred in the ice bath for $10 \mathrm{~min}$. A preliminary experiment revealed that the solution contained phenylacetaldehyde (PAA) and phenylacetonitrile (PAN) as well as NCPAAI. The solution underwent LLE with chloroform as the extractant. The chloroform extract was serially diluted (2-fold) with fresh chloroform, and benzaldehyde $(5 \mathrm{mg} / \mathrm{L})$ was added to each dilution and to the original extract as an internal standard. The serially diluted samples and the original extract were then analyzed by GCMS-O.

The third chlorinated solution, which was used to search for other TPs (i.e., TPs identified neither by P\&T - nor by LLE-GC/MS) and to evaluate their contributions to the off-flavor, was prepared as follows. Phosphate buffer $(100 \mathrm{mM}, \mathrm{pH} 7)$ was cooled in an ice bath, and enough sodium hypochlorite was added to achieve a concentration of $68.2 \mathrm{mg}-\mathrm{Cl}_{2} / \mathrm{L}$. Then phenylalanine $(200 \mu \mathrm{M})$ was added, and the resulting solution was stirred in the ice bath for $10 \mathrm{~min}$. The resulting solution (hereafter referred to as the TP search solution) was subjected SPME as described below with one modification: that is, the SPME fiber was exposed to the solution for $25,12.5,6.25,3.13,1.56,0.78$, and $0.39 \mathrm{~min}$ to prepare pseudo-serially diluted (2-fold) samples. The pseudo-serially diluted samples were analyzed by GC-MS-O.

\subsection{Conventional flask test}

The chlorinated phenylalanine-containing solution was subjected to a conventional flask test according to Standard Methods for the Examination of Water (Japan Water Works Association 2011) as follows (Fig. 2). The solution was serially diluted (2-fold) with phosphate buffer (0.1 mM, pH 7.0). Then $200-\mathrm{mL}$ aliquots of the original solution and each dilution were placed in Erlenmeyer flasks with ground glass stoppers. Each flask was grouped with two other flasks that contained only phosphate buffer (blanks) to form a dilution set. All flasks were preheated at $40^{\circ} \mathrm{C}$ in a water bath. The conventional flask test was performed by six assessors. For each dilution, a set of three flasks (two blanks and one containing the undiluted or diluted sample) was given to each assessor. In each set, the assessor attempted to identify which flask differed from the others. If the assessor successfully identified the sample flask twice for a given set, he or she tried the next set, and this process was continued until the assessor failed to identify the sample flask in a set. The fold-dilution value of the set just prior to the set at which the accessor failed was regarded as the off-flavor intensity. Finally, as the off-flavor intensity of the sample, we took the geometric mean of the off-flavor intensities determined by four assessors (the data for the most and least sensitive assessors were omitted).

To determine the odor thresholds of commercially available TPs [PAA, PAN, benzyl chloride (BC), chlorobenzene $(\mathrm{CB})$, benzaldehyde $(\mathrm{BA})$, and benzonitrile $(\mathrm{BN})]$, free chlorine, and laboratoryprepared trichloramine solution (Kosaka et al. 2010a), we prepared a standard solution of each compound, and then subjected these solutions to the conventional flask test after serial dilution as described in the preceding paragraph (Fig. 1). The geometric mean of the lowest concentrations at which four assessors perceived an off-flavor (the data for the most and least sensitive assessors were omitted) was taken as the odor threshold.

\subsection{Identification and quantification of TPs in the chlorinated phenylalanine-containing solution by means of $P \& T-G C / M S$}

To identify and quantify TPs generated from phenylalanine during chlorination, we performed P\&TGC/MS directly on the chlorinated phenylalanine-containing solution by using a gas chromatograph (7890A, Agilent Technologies, Inc., Palo Alto, CA, USA) and mass spectrometer (5975C, Agilent Technologies) coupled to a P\&T pretreatment system (Eclipse Purge-and-Trap Sample Concentrator, 
OI Analytical, Inc., College Station, TX, USA). The gas chromatograph was equipped with an HP$1 \mathrm{~ms}$ capillary column (length, $15 \mathrm{~m}$; internal diameter, $0.25 \mathrm{~mm}$; thickness, $0.25 \mu \mathrm{m}$; Agilent Technologies). GC/MS was first performed in scan mode, and the TPs were identified by database matching with National Information System for Science and Technology libraries and subsequent comparison of GC retention times and mass spectra with those of commercially available chemical standards of the TPs. The commercially available TPs were quantified by means of GC/MS in selected-ion-monitoring mode with anthracene as an internal standard; the fragment ions of PAA, BC, $\mathrm{BN}, \mathrm{BA}, \mathrm{CB}$, and PAN were detected at $\mathrm{m} / \mathrm{z}$ 91, 91, 103, 106, 112, and 117, respectively.

\subsection{Extraction methods}

\subsubsection{LLE}

Chloroform $(5 \mathrm{~mL})$ was added to $50 \mathrm{~mL}$ of each sample solution, and the mixture was vigorously shaken for 90 min with a mechanical shaker (SR-2 DS, Taitek Corp., Koshigaya, Japan). The chloroform phase was separated, dehydrated with anhydrous sodium sulfate, and then analyzed by GC-MS-O. BA served as an internal standard.

\subsubsection{SPME}

Sample solution $(10 \mathrm{~mL})$ was placed in a $20-\mathrm{mL}$ glass vial along with a magnetic stirrer. The vial was tightly sealed with a screw-top cap with a septum and then incubated at $40{ }^{\circ} \mathrm{C}$ in a water bath. An SPME fiber needle (PMDS/DVA; thickness, $65 \mu \mathrm{m}$; Sigma-Aldrich Japan, Tokyo, Japan) was inserted into the headspace of the vial through the septum; the needle was held there for 90 min, while the solution was vigorously stirred, to allow volatile TPs to be adsorbed by the SPME fiber. Then the SPME fiber was inserted into the headspace of a $20-\mathrm{mL}$ glass vial containing $\mathrm{BC}$ in $10 \mathrm{~mL}$ of Milli$\mathrm{Q}$ water (BC concentration, $5 \mathrm{mg} / \mathrm{L}$ ) and held there for $5 \mathrm{~min}$, while the liquid was vigorously stirred, to allow the fiber to adsorb $\mathrm{BC}$ as an internal standard. The $\mathrm{BC}$ concentration in the phenylalaninecontaining solution was expected to be negligible compared to the concentration of the $\mathrm{BC}$ standard solution. The fiber was then analyzed by GC-MS-O.

\section{$2.5 G C-M S-O$}

The GC-MS-O system consisted of a gas chromatograph (7890A, Agilent Technologies) and mass spectrometer (5975C, Agilent Technologies) coupled to a sniffing port (ODP 2, Gerstel GmbH \& Co. KG, Mülheim, Germany). The gas chromatograph was equipped with an HP-1ms capillary column (length, $15 \mathrm{~m}$; internal diameter, $0.25 \mathrm{~mm}$; thickness, $0.25 \mu \mathrm{m}$; Agilent Technologies). The undiluted chloroform extracts $(4 \mu \mathrm{L})$ obtained by LLE and the SPME fiber that had been exposed to the TP search solution for the longest time ( $25 \mathrm{~min}$; see sec. 2.1) were injected into the GC-MS-O system. The organic TPs (e.g., NCPAAI and PAA) in the samples were temporally separated by means of the capillary column of the gas chromatograph. At the outlet of the column, the gas flow was divided into two flows: one was introduced into the mass spectrometer to identify and quantify the organic compounds in the sample, and the other was introduced into the sniffing port for simultaneous and instantaneous determination of whether the compound was responsible for an off-flavor. If the assessor perceived an off-flavor from a TP, he or she assessed the next diluted or pseudo-diluted sample. This procedure was repeated until the assessor failed to perceive an off-flavor. For each diluted or pseudo-diluted sample, the GC/MS peak areas for NCPAAI, PAA, and other TPs for which the assessors perceived an off-flavor, and the internal standards, were recorded. GC-MS-O was performed with six assessors. The geometric mean of the intensities determined by four assessors (the data for the most and least sensitive assessors were omitted) was taken as the off-flavor intensity of each TP as determined by GC-MS-O.

2.6 Estimating the contributions of commercially unavailable TPs to the off-flavor of the chlorinated phenylalanine-containing solution by means of GC-MS-O combined with a conventional flask test The contributions of commercially unavailable TPs were estimated by combining GC-MS-O results 
with those of a conventional flask test.

For each commercially unavailable TP and for PAA, peak area ratios were determined for the highNCPAAI solution and the TP search solution by dividing the peak area of each compound by that of the internal standard. Then the relative concentration of each compound was calculated from the dilution ratio (or pseudo-dilution ratio), and the logarithm (base 10) of the relative concentration was plotted against the logarithm (base 10) of the peak area ratio. As an example, a plot of the PAA data generated by Assessor 1 is shown in Fig. 3. The relative concentration corresponding to the odor threshold of PAA for Assessor 1 ( $R C_{\text {OT,PAA, } 1)}$ was calculated from the regression line, as was the

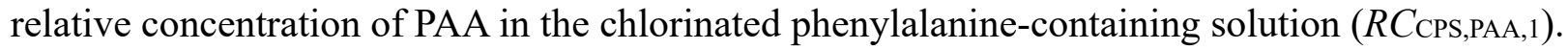

The off-flavor intensity of PAA in the chlorinated phenylalanine-containing solution as determined by GC-MS-O analysis by Assessor 1 (OFIGCMSO,PAA,1) was determined by means of the following equation:

$$
O F I_{\mathrm{GCMSO}, \mathrm{PAA}, 1}=\frac{R C_{\mathrm{CPS}, \mathrm{PAA}, 1}}{R C_{\mathrm{OT}, \mathrm{PAA}, 1}}
$$

The off-flavor intensity of each of the other commercially unavailable TPs as determined by GC-MS$\mathrm{O}$ analysis by Assessor $1\left(O F I_{\mathrm{GCMSO}, \mathrm{cuTP}, 1)}\right)$ was determined in the same manner. The off-flavor intensities were divided by the corresponding recovery ratios for LLE ( $R R_{\text {cuTP }}$ and $R R_{\text {PAA }}$ for each commercially unavailable TP and PAA, respectively) (Fig. S1), and then the off-flavor intensity of each commercially unavailable TP relative to that of PAA for Assessor 1 ( $\left.R O F I_{\text {cuTP, } 1}\right)$ was calculated:

$$
R O F I_{\text {cuTP }, 1}=\frac{O F I_{\mathrm{GCMSO}, \text { cuTP, } 1} / R R_{\mathrm{cuTP}}}{O F I_{\mathrm{GCMSO}, \mathrm{PAA}, 1} / R R_{\mathrm{PAA}}}
$$

The off-flavor intensity of each commercially unavailable TP relative to that of PAA (ROFI $\left.I_{\text {cuTP }}\right)$ was then calculated as the geometric mean of the $R O F I_{\text {cuTP, } i}$ values for four of the six assessors (the data for the most and least sensitive assessors were omitted from the calculations):

$$
R O F I_{\text {cuTP }}=\left(\prod_{i=1}^{4} R O F I_{\text {cuTP }, i}\right)^{1 / 4}
$$

Finally, the PAA-based off-flavor intensity of each commercially unavailable TP determined by means of the conventional flask test $\left(O F I_{\mathrm{FT}, \mathrm{cuTP}}\right)$ was estimated from the relative off-flavor intensity of the TP and the off-flavor intensity of PAA $\left(O F F_{\mathrm{FT}, \mathrm{PAA}}\right)$ in the flask test:

$$
O F I_{\mathrm{FT}, \text { cuTP }}=O F I_{\mathrm{FT}, \mathrm{PAA}} \times R O F I_{\text {cuTP }}
$$

\subsection{PAC treatment of chlorinated phenylalanine-containing solution}

Two types of PAC were used in the present study: (1) commercially available NPAC (Taiko W, Futamura Chemical Co., Nagoya, Japan), which has a median diameter of $8.0 \mu \mathrm{m}$ and is used in drinking water treatment plants, and (2) SPAC, which has a median diameter of $0.7 \mu \mathrm{m}$ and was prepared by pulverizing the NPAC with a wet bead mill (Metawater Co., Tokyo, Japan).

The chlorinated phenylalanine-containing solution was supplemented with $10 \mathrm{mg} / \mathrm{L}$ of either NPAC or SPAC, and the mixture was then stirred for $30 \mathrm{~min}$. Samples were withdrawn after 5 and $30 \mathrm{~min}$ of contact time and were filtered through a polytetrafluoroethylene membrane filter $(\phi=0.2 \mu \mathrm{m})$ to remove the PAC particles. The concentrations of TPs in the samples were then measured, and the offflavor intensities of the samples were evaluated by means of the conventional flask test.

To determine the mechanism by which PAC removed the off-flavor of the chlorinated phenylalaninecontaining solution, we treated a chlorinated ${ }^{15} \mathrm{~N}$-labeled-phenylalanine-containing solution with SPAC by using a procedure similar to that described by Sakuma et al. (2015) (see SI). The nitrogen mass balances before and after SPAC treatment were determined by measuring the concentrations of 
chloramines, PAN, and BA in the liquid phase and the concentration of ${ }^{15} \mathrm{~N}_{2}$ in the gas phase (see SI).

\section{Results and Discussion}

\subsection{Identification of TPs in the chlorinated phenylalanine-containing solution by means of P\&T- GC/MS}

No phenylalanine was detected in the chlorinated phenylalanine-containing solution, the indication being that the amino acid had been completely transformed into TPs by the chlorination. The P\&TGC/MS total ion chromatogram (TIC) of the solution (Fig. S3) exhibited six peaks that had not been observed prior to chlorination. A comparison of the mass spectrum of each peak with that in the National Information System for Science and Technology library suggested that the peaks corresponded to $\mathrm{CB}, \mathrm{BA}, \mathrm{BN}, \mathrm{BC}, \mathrm{PAA}$, and PAN. Comparisons of the mass spectra and the GC retention times of these peaks with the corresponding data for commercially available chemical standards confirmed these assignments (data not shown). PAA (Hrudey et al. 1988, Bruchet et al. 1992, Conyers and Scully 1993, Froese et al. 1999, Freuze et al. 2005, Ma et al. 2016), PAN (Conyers and Scully 1993, Conyers et al. 1993, Freuze et al. 2005, Ma et al. 2016), and BC (Ma et al. 2016) are reportedly produced during chlorination of phenylalanine. In contrast, $\mathrm{CB}, \mathrm{BA}$, and $\mathrm{BN}$ have not been reported to be TPs of phenylalanine, possibly because these compounds are produced in only very small amounts. Neither monochloramine nor dichloramine was detected in the chlorinated phenylalanine-containing solution, although trichloramine was detected. Our finding is consistent with that of Kosaka et al. (2010b), who also reported the production of trichloramine during chlorination of phenylalanine.

The major TPs of phenylalanine were PAA, PAN, and trichloramine (Fig. 4). The benzene rings of PAA and PAN accounted for $31 \%$ and $57 \%$, respectively, of the benzene ring content in the original phenylalanine. $\mathrm{CB}, \mathrm{BA}, \mathrm{BN}$, and $\mathrm{BC}$ were also detected by $\mathrm{GC} / \mathrm{MS}$, but only in small quantities. The TPs quantified in the present study accounted for $93 \%$ of the benzene ring content in the phenylalanine. Note that although the difference between the benzene ring contents before and after chlorination (i.e., 7\%) may be within experimental error (see SI for discussion of a possible statistical analysis of the difference), the difference may indicate the presence of TPs that were not detected by P\&T-GC/MS. Another possibility is that some of the benzene rings in aromatic compounds were cleaved during chlorination, generating chloroform (Dickenson et al. 2008, Gallard and von Gunten 2002) and chlorinated acetic acids (Dickenson et al. 2008).

In contrast, only $57 \%$ of the nitrogen atoms contained in the original phenylalanine could be accounted for by the quantified TPs: the major contributors were PAN (31\%) and trichloramine (25\%), leaving $43 \%$ of the nitrogen atoms unaccounted for. Phenylalanine may have been transformed into nitrogenous compounds that were not detected by our analytical methods. In addition, $\mathrm{N}_{2}$ gas may have been generated from ammonium nitrogen by means of breakpoint chlorination reactions between ammonium nitrogen and free chlorine and between monochloramine and dichloramine (Bauer and Snoeyink 1973). In the same manner, nitrogen atoms may have been released from the benzene ring as trichloramine and then transformed into $\mathrm{N}_{2}$ gas, which would have been released to the atmosphere, by means of breakpoint chlorination reactions. Experimental error also accounts for some of the missing nitrogen atoms.

3.2 Use of a conventional flask test to estimate the contributions of commercially available TPs to the off-flavor of the chlorinated phenylalanine-containing solution

The phenylalanine-containing solution had no off-flavor before chlorination. However, the chlorinated phenylalanine-containing solution had an average off-flavor intensity of 142 , as determined by means of the conventional flask test. To determine the contributions of the commercially available TPs to the off-flavor, we performed conventional flask tests on a solution of 
a chemical standard of each TP to determine its odor threshold. Every commercially available TP had a detectable odor (Table 1). The odor thresholds determined in the present study tended to be lower than previously reported thresholds, most likely because of differences in the procedures used for the sensory evaluation tests. The detected concentrations of PAA, PAN, trichloramine, and free chlorine in the chlorinated phenylalanine-containing solution were higher than the corresponding odor thresholds, whereas the concentrations of $\mathrm{BA}, \mathrm{CB}, \mathrm{BN}$, and $\mathrm{BC}$ were lower than the odor thresholds. This finding indicates that PAA, PAN, trichloramine, and free chlorine contributed to the off-flavor of the chlorinated phenylalanine-containing solution, whereas $\mathrm{BA}, \mathrm{CB}, \mathrm{BN}$, and $\mathrm{BC}$ did not.

To quantify the contributions of PAA, PAN, trichloramine, and free chlorine to the off-flavor of the chlorinated phenylalanine-containing solution, we calculated the off-flavor intensity of each of these four compounds by dividing its concentration in the chlorinated phenylalanine-containing solution by its odor threshold (Fig. 5). The sum of the calculated off-flavor intensities of these four compounds (63) was only $44 \%$ of the observed value (142), even though these compounds accounted for $93 \%$ of the benzene ring content of phenylalanine (Fig. 4). There are two possible explanations for this finding: (1) the off-flavors of these compounds may have interacted synergistically to increase the overall off-flavor intensity of the chlorinated phenylalanine-containing solution, and (2) TPs that were not detected by $\mathrm{P} \& \mathrm{~T}-\mathrm{GC} / \mathrm{MS}$ may have contributed to the off-flavor.

To investigate the possibility of synergism, we prepared a solution of PAN + PAA + free chlorine and a solution of PAN + PAA + free chlorine + trichloramine at concentrations that were the same as the corresponding concentrations in the chlorinated phenylalanine-containing solution; then we subjected these solutions to the conventional flask test. The observed off-flavor intensities of these solutions were almost the same as the calculated intensities (Fig. S5), the indication being that the contributions of these TPs to the observed off-flavor were additive rather than synergistic. Olfactometric interactions among odorants in mixtures remain a topic of debate but are customarily divided into three types: (1) hypoadditive, in which the odor intensity of a mixture is less than the sum of the odor intensities of its constituents (Olsson 1994, Cain et al. 1995); (2) completely additive, in which the odor intensity of a mixture is equal to the sum of the odor intensities of its constituents (Patterson et al. 1993, Wise and Cain 2000); and (3) hyperadditive, in which the odor intensity of a mixture is greater than the sum of the odor intensities of its constituents (Laska and Hudson 1991, Miyazawa et al. 2008). In our case, the interactions between PAN, PAA, free chlorine, and trichloramine exhibited complete additivity. At the molecular level, an odorant is perceived by a distinct combination of odorant receptors located on the olfactory epithelium (Kajiya et al. 2001). Odorant receptors not only discriminate subtle differences between the chemical structures of odorants but also recognize groups of odorants with similar molecular structures (Kajiya et al. 2001). Thus, the structural similarity between PAN and PAA might explain the complete additivity that we observed, but the reason for the complete additivity of PAN, PAA, free chlorine, and trichloramine was unclear because the structures of the latter two compounds differ considerably from those of PAN and PAA. Nevertheless, the complete additivity suggests that the difference between the observed and calculated off-flavor intensities was most likely due to the contributions of undetected TPs.

\subsection{Detection of NCPAAI odor by LLE-GC-MS-O}

Although NCPAAI is reportedly generated from phenylalanine during chlorination, we did not detect it by $\mathrm{P} \& \mathrm{~T}-\mathrm{GC} / \mathrm{MS}$ of the chlorinated phenylalanine-containing solution. To determine whether NCPAAI was in fact produced from phenylalanine in this study, we conducted an analysis in which the chlorinated phenylalanine-containing solution was pretreated by LLE with chloroform rather than by P\&T prior to GC/MS. Three peaks were detected in the resulting GC/MS TIC (Fig. 6a). Peaks 61 and 6-2 were assigned to PAA and PAN, respectively, by comparison of their mass spectra and GC retention times with those of commercially available chemical standards. Peak 6-3 was determined to be NCPAAI by analysis of its mass spectrum (Fig. S6). Although Freuze et al. (2005) reported that 
NCPAAI contributed to the off-flavor of a chlorinated phenylalanine solution and estimated its odor threshold to be $3 \mu \mathrm{g} / \mathrm{L}$, as indicated by a conventional flask test conducted on a high-NCPAAI solution under the assumption that $35 \%$ of the initially added phenylalanine had been transformed to NCPAAI, these investigators did not discuss the basis for this assumption. Accordingly, whether this compound contributes to off-flavor and what its odor threshold may be remain unclear.

To determine whether NCPAAI contributed to the off-flavor of the chlorinated phenylalaninecontaining solution in this study, we analyzed a high-NCPAAI solution by means of GC-MS-O (Fig. $6 b)$. Again, we observed three peaks in the GC/MS TIC, and they had the same retention times as the peaks observed for the chlorinated phenylalanine-containing solution. An off-flavor was perceived three times during the olfactory portion of the analysis, and these times corresponded to the retention times of PAA, PAN, and NCPAAI (Peaks 6-1-6-3, respectively). These findings clearly indicate that NCPAAI, as well as PAA and PAN, contributed to the off-flavor.

\subsection{Use of SPME-GC-MS-O to identify other TPS that contributed to the off-flavor of the chlorinated phenylalanine-containing solution}

To search for TPs that were not detected by means of P\&T-GC/MS or LLE-GC/MS, we used SPME as a pretreatment method prior to GC/MS. Several peaks appeared in the resulting GC/MS TIC (Fig. 7a). Peak 7-1 corresponded to BC, which had been added to the solution as an internal standard; and Peaks 7-2 and 7-3 corresponded to PAA and PAN, respectively. An off-flavor was perceived twice during the olfactory portion of the analysis (Fig. 7b): once at a retention time of approximately 3.6 min, which corresponded to PAA (Peak 7-2), and once at a retention time of approximately $6.2 \mathrm{~min}$, which corresponded to Peak 7-4. These findings suggest that the TP corresponding to Peak 7-4 most likely contributed the overall off-flavor of the chlorinated phenylalanine-containing solution. On the basis of MS (Fig. S7 and Table S1), we assigned Peak 7-4 to 2-chloro-2-phenylacetaldehyde (2C2PAA), which has not previously been reported to be produced during chlorination of phenylalanine.

\subsection{Contributions of NCPAAI and 2C2PAA to the off-flavor of the chlorinated phenylalanine- containing solution}

Because chemical standards for NCPAAI and 2C2PAA are not commercially available, we could not perform a conventional flask test on these two TPs or evaluate their odor thresholds. Accordingly, their contributions to the off-flavor of the chlorinated phenylalanine-containing solution could not be evaluated by means of a conventional flask test alone. Instead, we estimated their contributions by combining the results of GC-MS-O with those of a conventional flask test (sec. 2.6).

In this way, we calculated the relative off-flavor intensities of NCPAAI to be 0.21 , whereas the offflavor intensity of PAA in the conventional flask test was 16. Using Eq. 4, we then calculated the offflavor intensity of NCPAAI in the flask test to be 3 , which accounted for $2 \%$ of the overall off-flavor of the chlorinated phenylalanine-containing solution. Similarly, the relative off-flavor intensity of $2 \mathrm{C} 2 \mathrm{PAA}$ in the flask test was calculated to be 1.1 , and the off-flavor intensity of 2C2PAA was calculated to be 18 , which accounted for $13 \%$ of the off-flavor. In summary, GC-MS-O combined with the conventional flask test showed that approximately $60 \%$ of the off-flavor generated during chlorination of phenylalanine could be accounted by free chlorine (13\%), 2C2PAA (13\%), trichloramine (12\%), PAA (11\%), PAN (8\%), and NCPAAI (2\%).

GC-MS-O allowed us to identify off-flavor compounds that were impossible to evaluate individually because they were part of a mixture. This method was thus revealed to be a powerful tool for evaluating the contribution of individual compounds to off-flavor, regardless of their commercial availability. Combining GC-MS-O and with the conventional flask test allowed us to identify TPs responsible for approximately $60 \%$ of the off-flavor of the chlorinated phenylalanine-containing 
solution. However, $40 \%$ of the off-flavor remained unexplained and was probably due to odorous TPs that were not detected by any of the analytical methods we used. Other extraction methods will be necessary to identify these other sources of off-flavor.

\subsection{PAC treatment of the chlorinated phenylalanine-containing solution}

We evaluated the effects of PAC treatment on the off-flavor intensity of the chlorinated phenylalaninecontaining solution (Fig. 8); 2C2PAA was not included in this evaluation, because it was performed before we had established that 2C2PAA contributed to the off-flavor. Treatment with NPAC gradually decreased the off-flavor intensity: after $30 \mathrm{~min}$ of NPAC contact time, the original intensity had decreased by $83 \%$. In contrast, treatment with SPAC for only 5 min decreased the off-flavor intensity to a value lower than that achieved with NPAC after $30 \mathrm{~min}$; furthermore, after $30 \mathrm{~min}$ of SPAC contact time, the off-flavor intensity had decreased by $95 \%$. These results indicate that PAC effectively removed off-flavor compounds from the chlorinated phenylalanine-containing solution and that increasing the PAC surface area by means of pulverization increased its effectiveness. PAC treatment removed both off-flavor derived from the identified TPs and that derived from unidentified TPs.

To investigate the mechanism by which SPAC removed the off-flavor compounds, we chlorinated a solution of ${ }^{15} \mathrm{~N}$-labeled phenylalanine and then measured the amounts of ${ }^{15} \mathrm{~N}$ before and after SPAC treatment (Fig. S8). Before SPAC treatment, the amount of ${ }^{15} \mathrm{~N}$ in the quantifiable TPs (i.e., BN, PAN, and trichloramine) in the liquid phase was $3.3 \mu \mathrm{g}, 0.58 \mu \mathrm{g}$ of which was contained in trichloramine. After SPAC treatment, the amount of ${ }^{15} \mathrm{~N}$ in the liquid phase decreased to $0.4 \mu \mathrm{g}$, all of which was attributable to the residual PAN. In the gas phase, we detected ${ }^{15} \mathrm{~N}_{2}$ in an amount equivalent to 0.54 $\mu \mathrm{g}$ of ${ }^{15} \mathrm{~N}$ that was present in trichloramine in the liquid phase before SPAC treatment. This observation suggests that trichloramine was transformed to $\mathrm{N}_{2}$ gas by reductive reactions mediated by functional groups on the surface of the SPAC particles (Sakuma et al. 2015). The other organic nitrogens were most likely not transformed into $\mathrm{N}_{2}$ gas but rather were adsorbed onto the carbon particles. In addition, nitrogenous TPs may have been converted to TPs that did not contribute to the off-flavor, although this possibility is unlikely. The removal of the off-flavor by treatment with activated carbon appeared to be due mainly to the reductive decomposition of trichloramine into $\mathrm{N}_{2}$ gas and probably also to the adsorption of the other TPs. Finally, whereas both PAA and NCPAAI were completely removed by treatment with SPAC, probably via adsorption, PAN was detectable even after treatment (Figs. 8 and S8). The hydrophilicity of a compound is known to influence its adsorption onto activated carbon particles: the tendency of a compound to be adsorbed by PAC decreases with decreasing $\log K_{\text {ow }}$ (i.e., with increasing hydrophilicity). Because the $\log K_{\text {ow }}$ of PAN (1.56, Hansch and Leo 1995) is smaller than the values for PAA (1.78, Hansch and Leo 1995) and NCPAAI (2.23, predicted by Marvin Sketch ver. 17.1.9.0, ChemAxon, Budapest, Hungary), PAN was probably less efficiently adsorbed by the SPAC particles than were the other two compounds.

\section{Conclusions}

1. The analytical method used in the present study, that is, a conventional flask test combined with GC-MS-O, proved to be a powerful tool for identifying compounds responsible for the offflavor generated by chlorination of aqueous phenylalanine.

2. GC-MS-O combined with pretreatment by means of LLE or SPME, respectively, revealed that NCPAAI and 2C2PAA, which are not commercially available and therefore cannot be assessed by means of conventional sensory evaluation tests, contributed to the off-flavor.

3. The combination of GC-MS-O and a conventional flask test indicated that approximately $60 \%$ of the off-flavor of the chlorinated phenylalanine-containing solution could be accounted for by free chlorine $(13 \%), 2 \mathrm{C} 2 \mathrm{PAA}(13 \%)$, trichloramine $(12 \%)$, PAA $(11 \%)$, PAN (8\%), and NCPAAI $(2 \%)$. 
4. Treatment with PAC removed the off-flavor of the chlorinated phenylalanine-containing solution. Experiments involving chlorination of aqueous ${ }^{15} \mathrm{~N}$-labeled phenylalanine revealed that the off-flavor was likely removed by reductive decomposition of trichloramine into $\mathrm{N}_{2}$ gas and by adsorption of the other TPs onto the carbon particles. The removal efficiency was enhanced by pulverization of the PAC.

\section{Acknowledgements}

This research was supported in part by a Grant-in-Aid for Scientific Research (S) (no. 24226012) from the Japan Society for the Promotion of Science; by a Grant-in-Aid for Challenging Exploratory Research (no. 15K12250) from the Japan Society for the Promotion of Science; by a Health and Labor Sciences Research Grant (Research on Health Security Control) from the Ministry of Health, Labor, and Welfare of Japan; and by the Bureau of Waterworks, Tokyo Metropolitan Government.

\section{References}

American Public Health Association/American Water Works Association/Water Environment Federation (2005) Standard Methods for Examination of Water and Wastewater, American Public Health Association.

Amoore, J. E. and Hautala, E. (1983) Odor as an aid to chemical safety: odor thresholds compared with threshold limit values and volatilities for 214 industrial chemicals in air and water dilution. Journal of Applied Toxicology 3(6), 272-290.

Bauer, R.C. and Snoeyink, V.L. (1973) Reactions of chloramines with active carbon. Journal Water Pollution Control Federation 45(11), 2290-2301.

Benanou, D., Acobas, F., de Roubin, M.R., David, F. and Sandra, P. (2003) Analysis of off-flavors in the aquatic environment by stir bar sorptive extraction-thermal desorption-capillary GC/MS/olfactometry. Analytical and Bioanalytical Chemistry 376(1), 69-77.

Bruchet, A., Costentin, E., Legrand, M.F. and Mallevialle, J. (1992) Influence of the chlorination of natural nitrogenous organic compounds on tastes and odors in finished drinking waters. Water Science and Technology 25(2), 323-333.

Buttery, R. G., Turnbaugh, J. G. and Ling, L. C. (1988) Contribution of volatiles to rice aroma. Journal of Agricultural and Food Chemistry 36, 1006-1009.

Cain, W.S., Schiet, F.T., Olsson, M.J. and de Wijk, R.A. (1995) Comparison of Models of Odor Interaction. Chemical Senses 20(6), 625-637.

Conyers, B. and Scully, F.E. (1993) N-chloroaldimines. 3. Chlorination of phenylalanine in model solutions and in a wastewater. Environmental Science \& Technology 27(2), 261-266.

Conyers, B., Walker, E., Scully, F.E. and Marbury, G.D. (1993) N-chloroaldimines. 4. Identification in a chlorinated municipal wastewater by gas chromatography/mass spectrometry. Environmental Science \& Technology 27(4), 720-724.

Delahunty, C.M., Eyres, G. and Dufour, J.-P. (2006) Gas chromatography-olfactometry. Journal of Separation Science 29(14), 2107-2125.

Dickenson, E.R.V., Summers, R.S., Croué, J.P. and Gallard, H. (2008) Haloacetic acid and trihalomethane formation from chlorination and bromination of aliphatic $\beta$-dicarbonyl acid model compounds. Environmental Science \& Technology 42, 3226-233.

Eduardo, I., Chietera, G., Bassi, D., Rossinib, L. and Vecchiettia, A. (2010) Identification of key odor volatile compounds in the essential oil of nine peach accessions. Journal of the Science of Food and Agriculture 90, 1146-1154.

Freuze, I., Brosillon, S., Herman, D., Laplanche, A., Démocrate, C. and Cavard, J. (2004) Odorous Products of the Chlorination of Phenylalanine in Water: Formation, Evolution, and Quantification. Environmental Science \& Technology 38(15), 4134-4139.

Freuze, I., Brosillon, S., Laplanche, A., Tozza, D. and Cavard, J. (2005) Effect of chlorination on the 
formation of odorous disinfection by-products. Water Res 39(12), 2636-2642.

Froese, K.L., Wolanski, A. and Hrudey, S.E. (1999) Factors governing odorous aldehyde formation as disinfection by-products in drinking water. Water Res 33(6), 1355-1364.

Gallard, H. and von Gunten, U. (2002) Chlorination of phenols: kinetics and formation of chloroform. Environmental Science \& technology 36, 884-890.

Hansch, C. and Leo, A. (1995) Exploring QSAR, American Chemical Society, Washington.

Hochereau, C. and Bruchet, A. (2004) Design and application of a GC-SNIFF/MS system for solving taste and odour episodes in drinking water. Water Science and Technology 49(9), 81-87.

Hrudey, S.E., Gac, A. and Daignault, S.A. (1988) Potent odour-causing chemicals arising from drinking water disinfection. Water Science and Technology 20(8-9), 55-61.

Japan Water Works Association (2011) Standard Methods for the Examination of Water.

Kajino, M., Morizane, K., Umetani, T. and Terashima, K. (1999) Odors arising from ammonia and amino acids with chlorine during water treatment. Water Science and Technology 40(6), 107-114.

Kajiya, K., Inaki, K., Tanaka, M., Haga, T., Kataoka, H. and Touhara, K. (2001) Molecular Bases of Odor Discrimination: Reconstitution of Olfactory Receptors that Recognize Overlapping Sets of Odorants. The Journal of Neuroscience 21(16), 6018-6025.

Kosaka, K., Seki, K., Kimura, N., Kobayashi, Y. and Asami, M. (2010a) Determination of trichloramine in drinking water using headspace gas chromatography/mass spectrometry. Water Science and Technology: Water Supply 10(1), 23-29.

Kosaka, K., Suzuki, K., Itoh, T., Echigo, S., Asami, M. and Akiba, M. (2010b) Characteristics of trichloramine formation by chlorination of amino acids. Environmental Engineering Research 47, 15-20 (in Japanese).

Krasner, S. W. and Barrett, S. E. (1984) Aroma and flavor characteristics of free chlorine and chloramines. Proceedings of the 12th Annual American Water Works Association Water Quality Technology, Denver, 381-398.

Laska, M. and Hudson, R. (1991) A comparison of the detection thresholds of odour mixtures and their components. Chemical Senses 16(6), 651-662.

Ma, X., Deng, J., Feng, J., Shanaiah, N., Smiley, E. and Dietrich, A.M. (2016) Identification and characterization of phenylacetonitrile as a nitrogenous disinfection byproduct derived from chlorination of phenylalanine in drinking water. Water Res 102, 202-210.

Miyazawa, T., Gallagher, M., Preti, G. and Wise, P.M. (2008) Synergistic mixture interactions in detection of perithreshold odors by humans. Chemical Senses 33, 363-369.

Noguerol-Pato, R., González-Rodríguez, R. M., González-Barreiro, C., Cancho-Grande, B. and Simal-Gándara, J. (2011) Influence of tebuconazole residues on the aroma composition of Mencía red wines. Food Chemistry 124, 1525-1532.

Noguerol-Pato, R., González-Álvarez, M., González-Barreiro, C., Cancho-Grande, B. and SimalGándara, J. (2013) Evolution of the aromatic profile in Garnacha Tintorera grapes during raisining and comparison with that of the naturally sweet wine obtained. Food Chemistry 139, 1052-1061.

Olsson, M.J. (1994) An interaction model for odor quality and intensity. Perception \& Psychophysics 55(4), 363-372.

Patterson, M.Q., Stevens, J.C., Cain, W.S. and Cometto-Muñiz, J.E. (1993) Detection thresholds for an olfactory mixture and its three constituent compounds. Chemical Senses 18(6), 723-734.

Peter, A., Köster, O., Schildknecht, A. and von Gunten, U. (2009) Occurrence of dissolved and particle-bound taste and odor compounds in Swiss lake waters. Water Res 43(8), 2191-2200.

Piriou, P., Mackey, E. D., Suffet, I. H. and Bruchet, A. (2004) Chlorinous flavor perception in drinking water. Water Science and Technology 49(9), 321-328.

Sakuma, M., Matsushita, T., Matsui, Y., Aki, T., Isaka, M. and Shirasaki, N. (2015) Mechanisms of trichloramine removal with activated carbon: Stoichiometric analysis with isotopically labeled trichloramine and theoretical analysis with a diffusion-reaction model. Water Res 68(0), 839-848.

Wise, P.M. and Cain, W.S. (2000) Latency and accuracy of discriminations of odor quality between binary mixtures and their components. Chemical Senses 25, 247-265. 


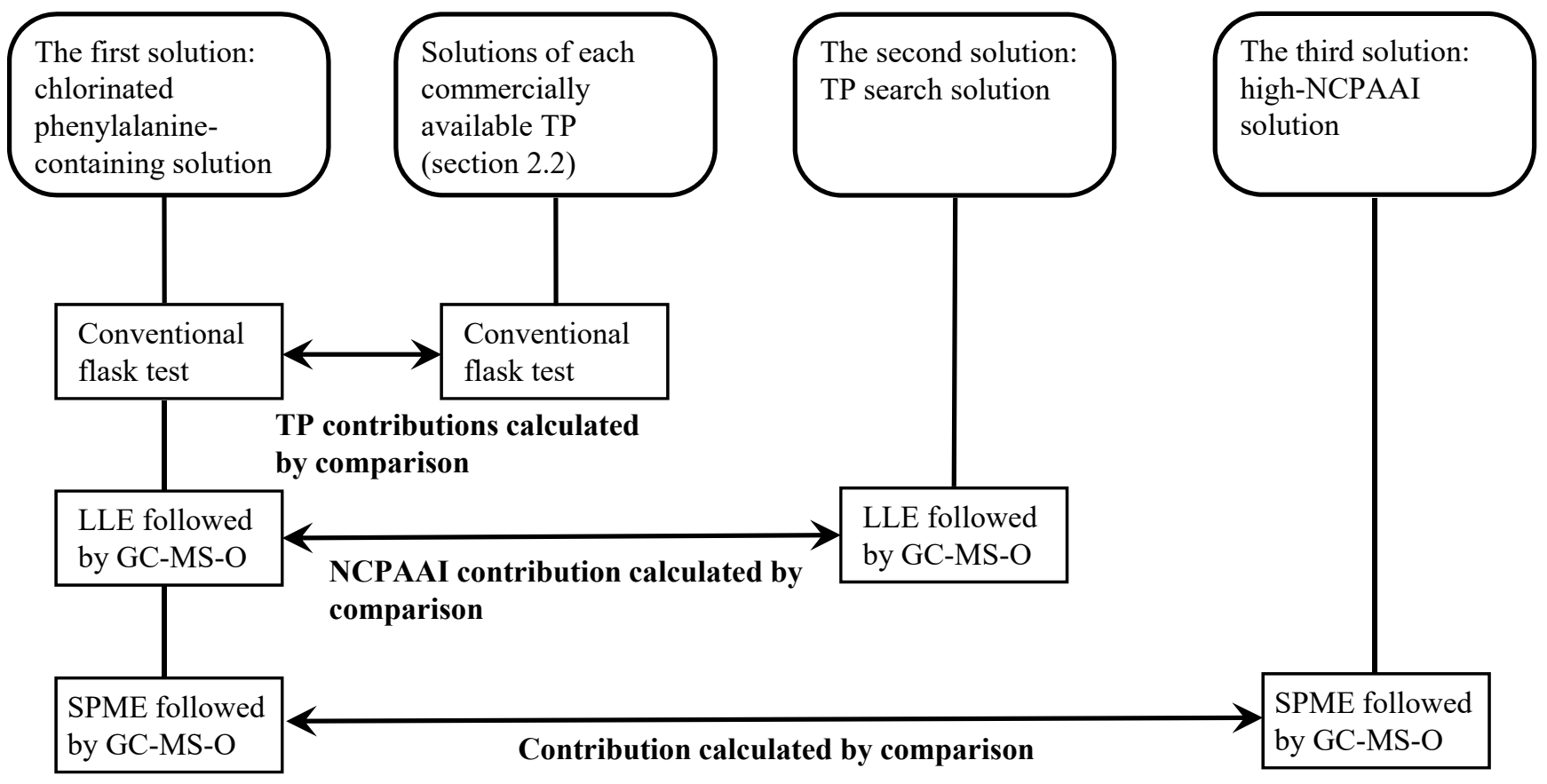

Fig. 1 Conceptual diagram of the procedure for elucidating the contributions of formed TPs to the off-flavor of the chlorinated phenylalanine-containing solution. LLE, liquid/liquid extraction; SPME, solid-phase microextraction; GCMS-O, gas chromatography-mass spectrometry-olfactometry; P\&T-GC/MS, purge and trap-gas chromatography-mass spectrometry; TP, transformation product; NCPAAI, $N$-chlorophenylacetaldimine. 


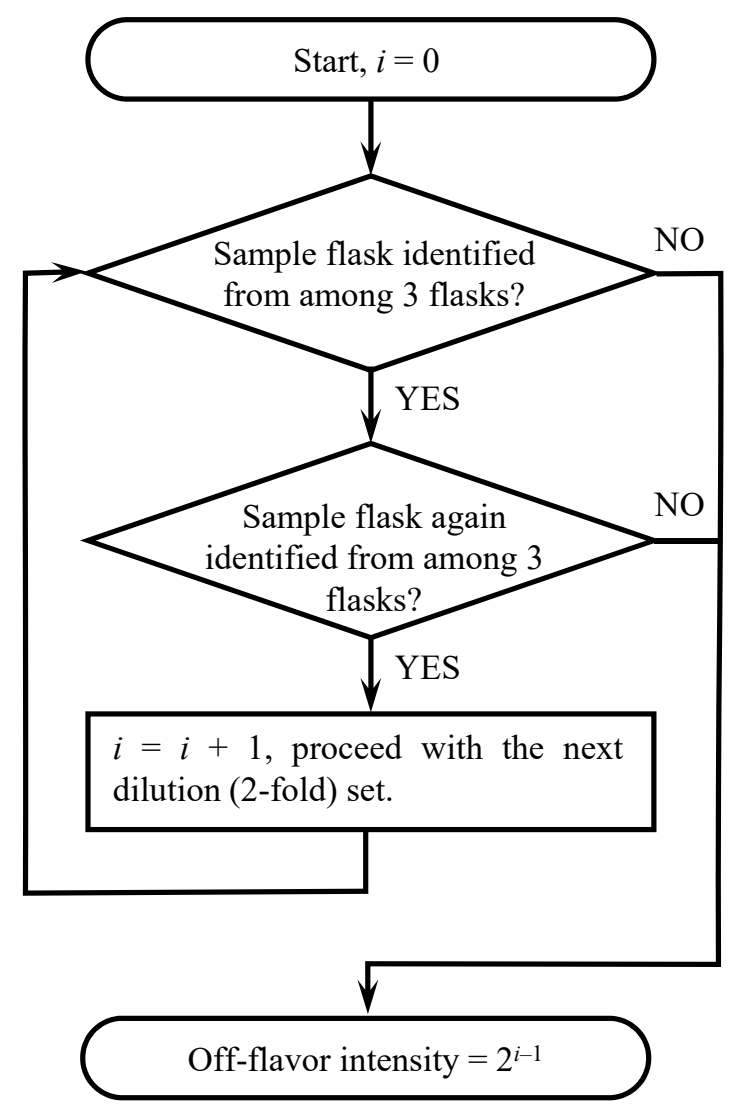

Fig. 2 Flowchart of conventional flask test. 


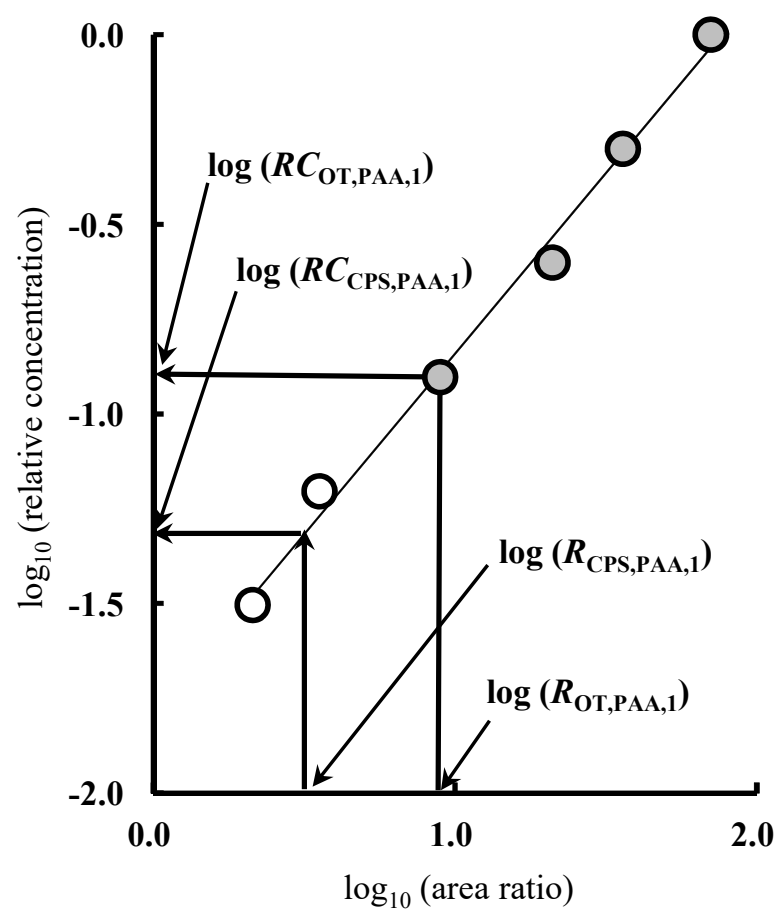

Fig. 3 Example of logarithmic relationship between the relative concentration and the area ratio of PAA (phenylacetaldehyde) obtained in the sensory evaluation test with the GC-MS-O analysis for Assessor 1. Gray circles represent data points at which the assessor perceived off-flavor; white circles represent data points at which the assessor perceived no off-flavor. $R_{\mathrm{CPS}, \mathrm{PAA}, 1}$ and $R_{\mathrm{OT}, \mathrm{PAA}, 1}$ represent the logarithm to the base 10 of the area ratio of PAA observed for the chlorinated phenylalanine-containing solution and the most dilute sample of the high NCPAAI solution from which the assessor perceived the off-flavor of PAA (i.e., the odor threshold), respectively. 




Fig. 4 TPs generated during chlorination of the phenylalaninecontaining solution. PAA, phenylacetaldehyde; PAN, phenylacetonitrile; $\mathrm{NCl}_{3}$, trichloramine; $\mathrm{BA}$, benzaldehyde; $\mathrm{CB}$, chlorobenzene; $\mathrm{BN}$, benzonitrile; $\mathrm{BC}$, benzyl chloride. 


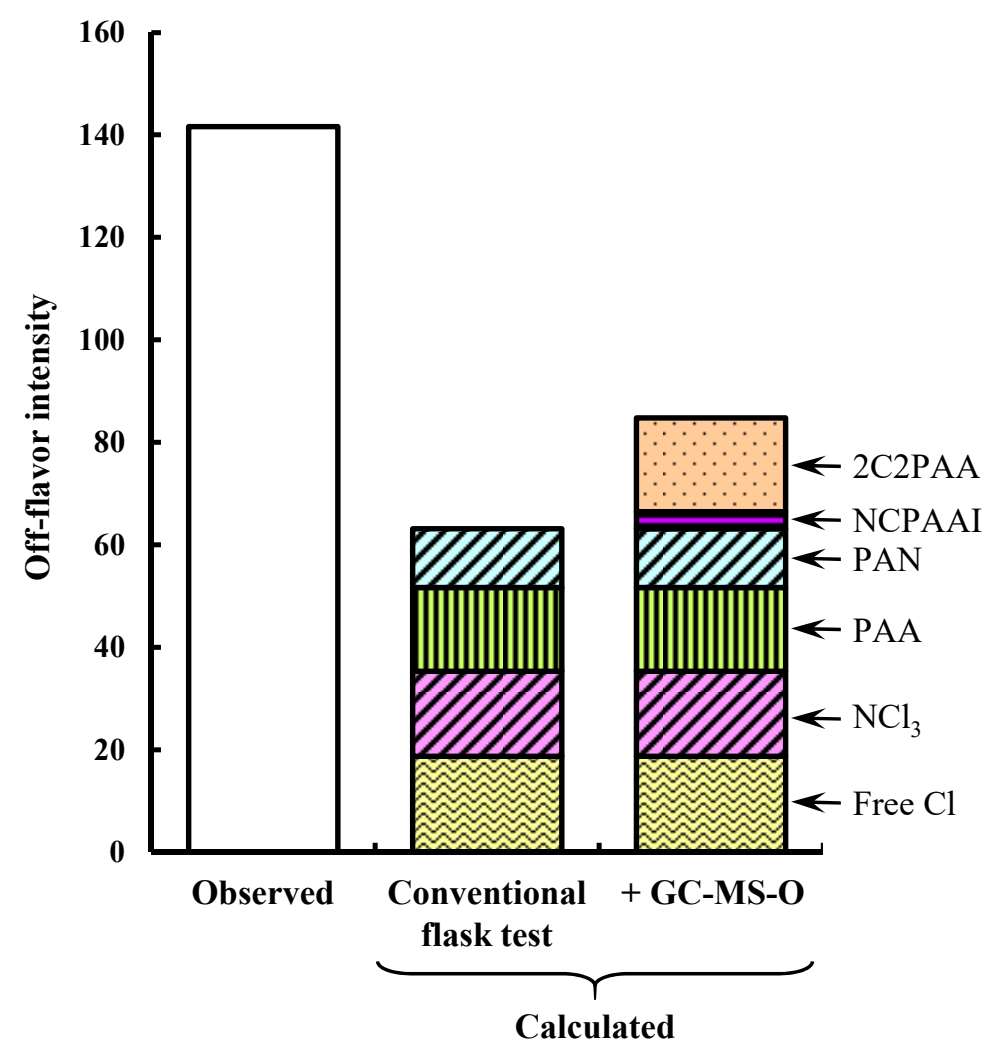

Fig. 5 Comparison of observed and calculated off-flavor intensities of the chlorinated phenylalanine-containing solution. The calculated value for the "Conventional flask test" was determined from the results of the sensory evaluation with the conventional flask test, whereas that for "+ GC-MS-O" was determined from the combined results of the conventional flask test and the GC-MS-O analysis. 2C2PAA, 2-chloro-2-phenylacetaldehyde; NCPAAI, $N$ chlorophenylacetaldimine; PAN, phenylacetonitrile; PAA, phenylacetaldehyde; $\mathrm{NCl}_{3}$, trichloramine; free $\mathrm{Cl}$, free chlorine. 

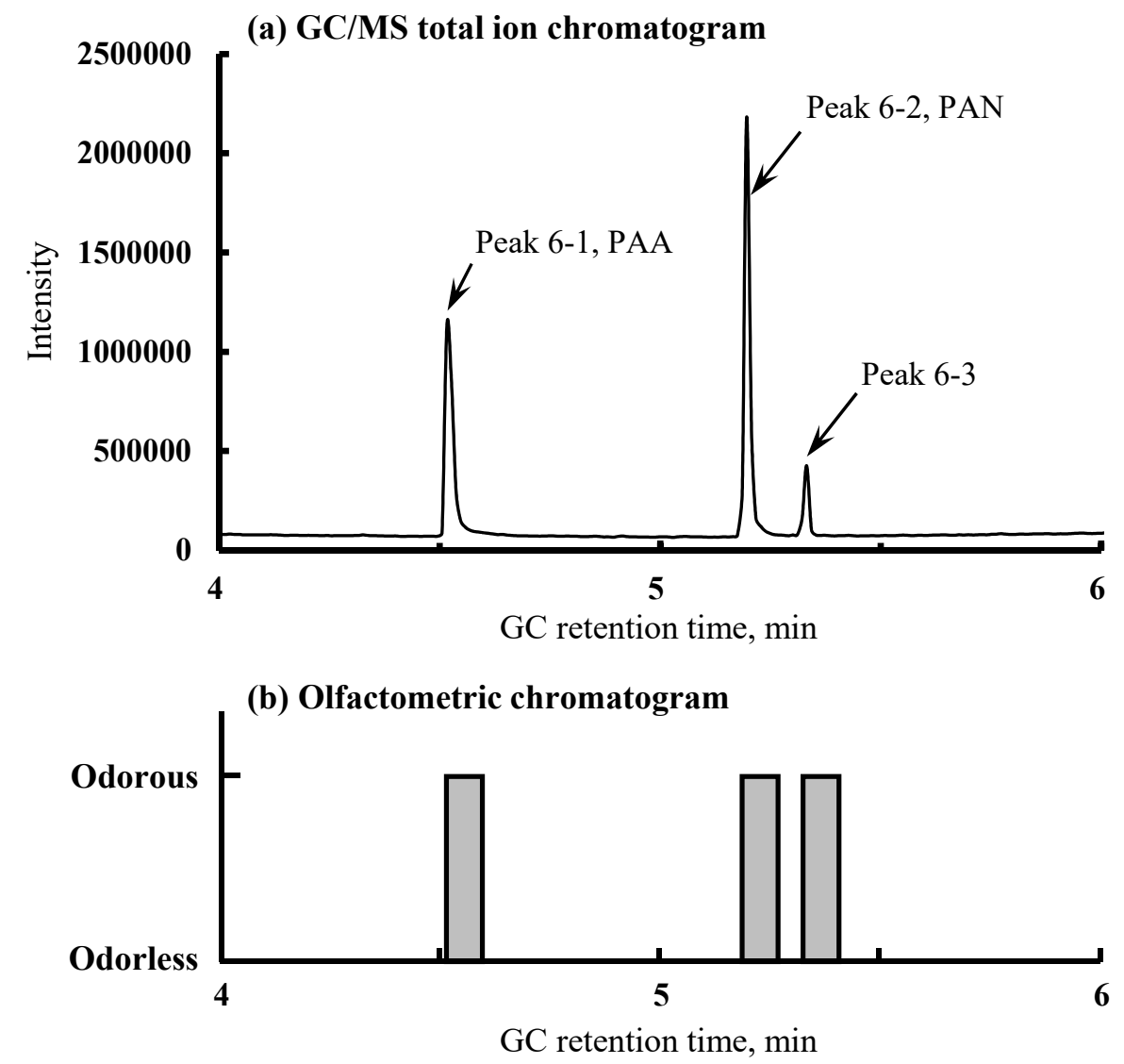

Fig. 6 (a) GC/MS total ion chromatogram of the chlorinated phenylalaninecontaining solution after the LLE with chloroform and (b) olfactometric chromatogram of the high-NCPAAI solution after the LLE with chloroform. PAA, phenylacetaldehyde; PAN, phenylacetonitrile. 

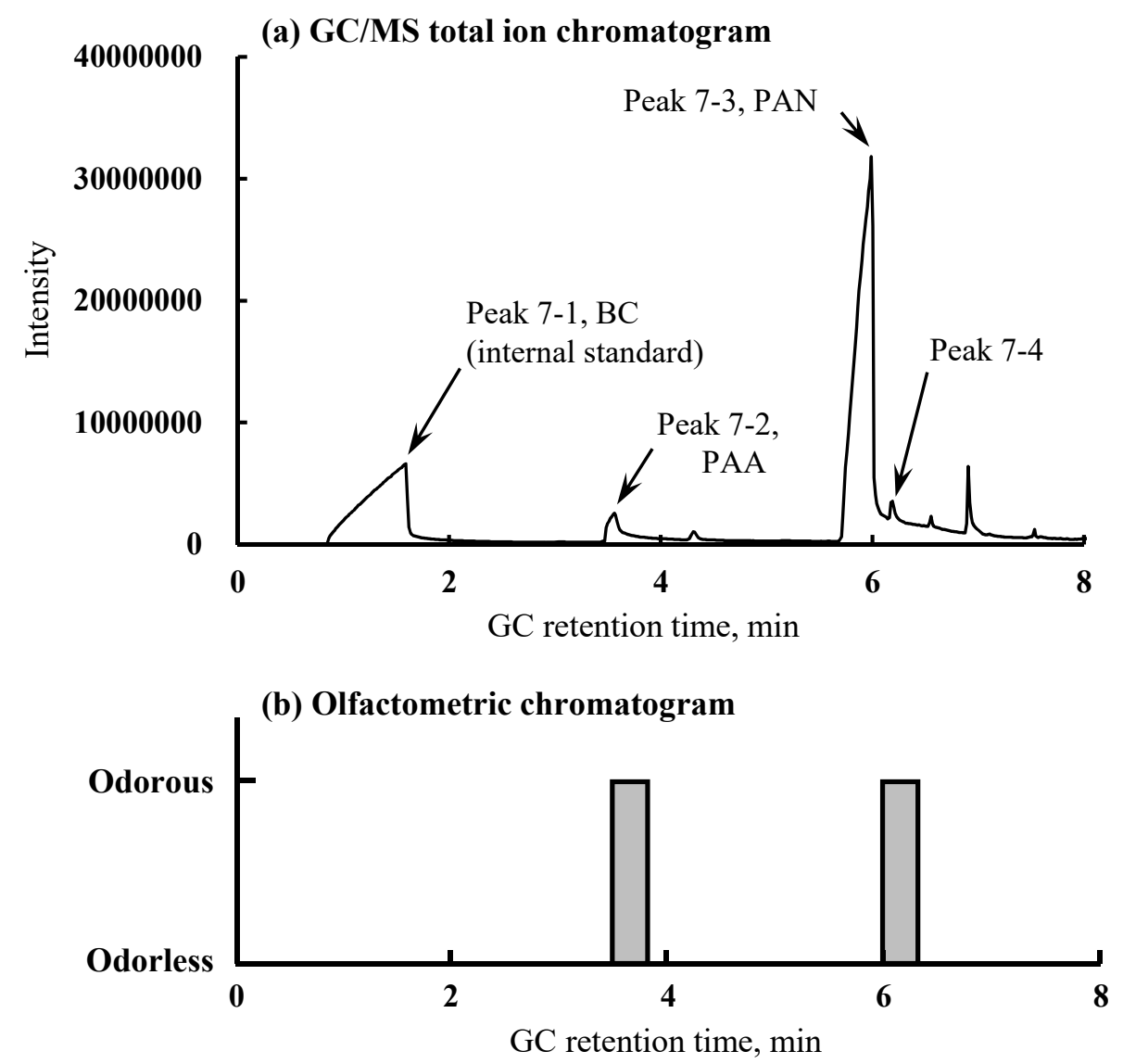

Fig. 7 (a) GC/MS total ion chromatogram and (b) olfactometric chromatogram of the TP search solution after the SPME. BC, benzyl chloride (internal standard); PAA, phenylacetaldehyde; PAN, phenylacetonitrile. 


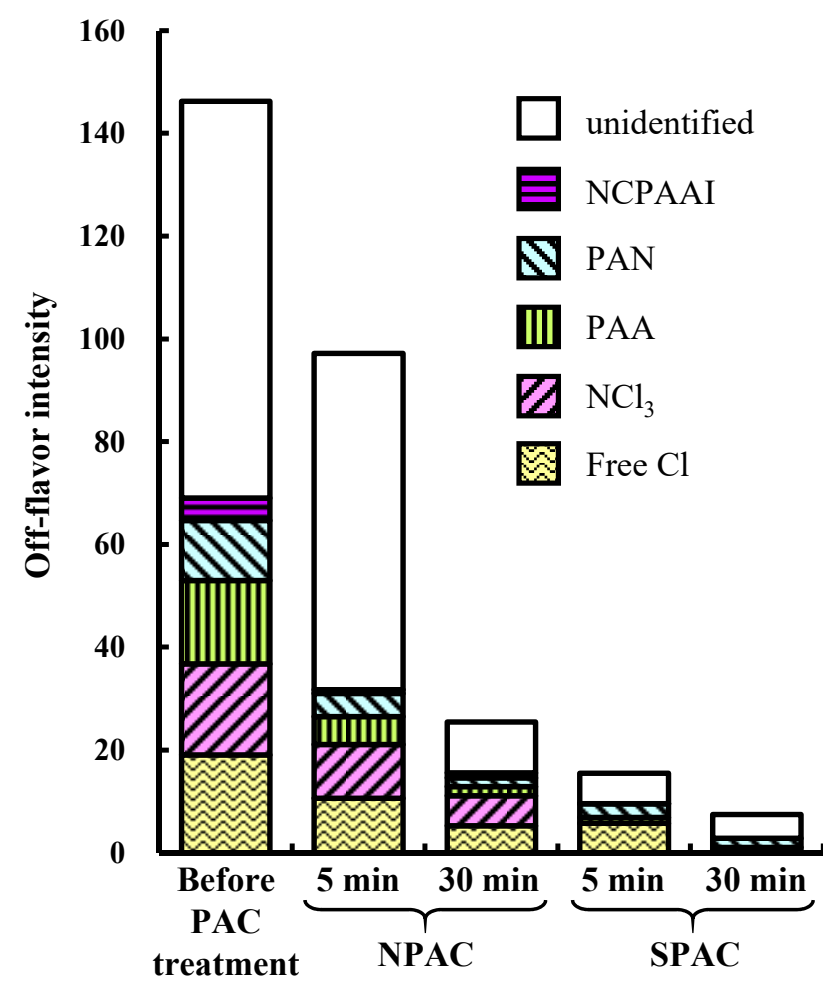

Fig. 8 Change in off-flavor intensity of the chlorinated phenylalanine-containing solution during PAC treatment. NCPAAI, $N$-chlorophenylacetaldimine; PAN, phenylacetonitrile; PAA, phenylacetaldehyde; $\mathrm{NCl}_{3}$, trichloramine; free $\mathrm{Cl}$, free chlorine. 


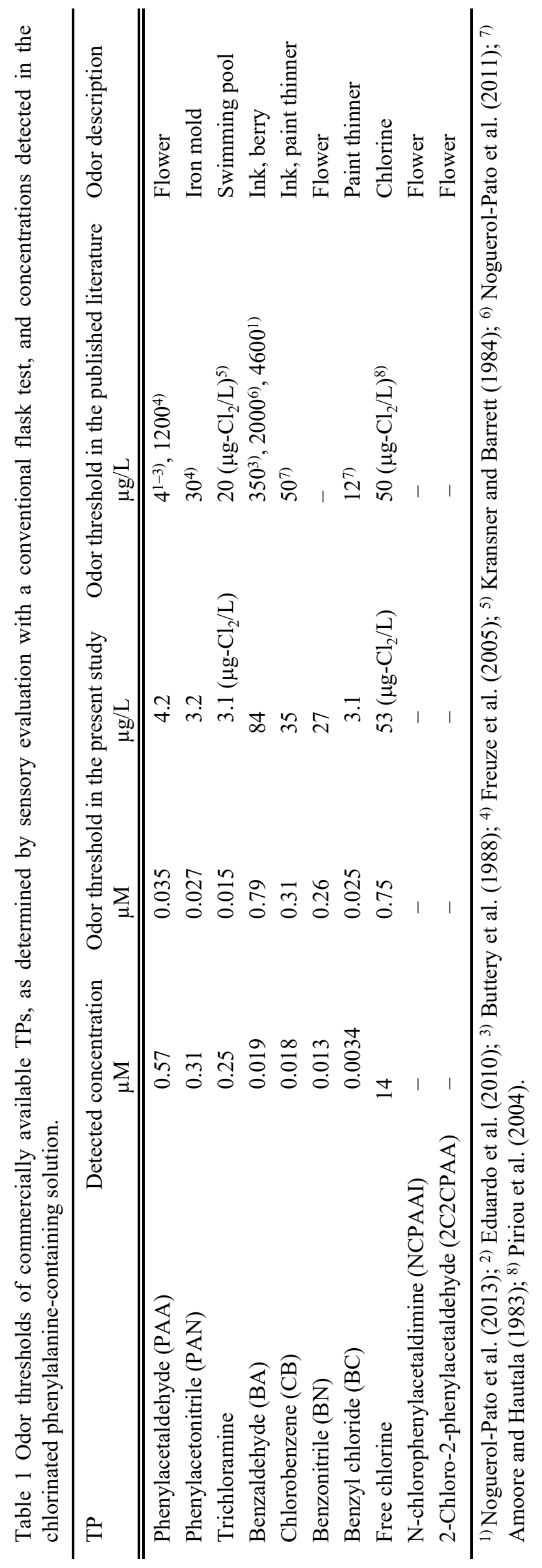




\title{
Supplementary information
}

\section{Use of gas chromatography-mass spectrometry-olfactometry and a conventional flask test to identify off-flavor compounds generated from phenylalanine during chlorination of drinking water}

\author{
Taku Matsushita*, Miki Sakuma, Shiori Tazawa, Taiki, Hatase, \\ Yoshihiko Matsui and Nobutaka Shirasaki
}

Graduate School of Engineering, Hokkaido University, N13W8, Sapporo 060-8628, Japan

*Corresponding author: e-mail, taku-m@eng.hokudai.ac.jp; Tel/Fax, +81-11-706-7279

\section{Quantification of phenylalanine by use of LC/MS/MS analysis}

Phenylalanine was directly quantified by using a liquid chromatography-electrospray-ionizationtandem mass spectrometer (LC-ESI/MS/MS, API3000, Applied Biosystems, Foster City, CA, USA) without extraction. Separation was achieved with a reverse-phase column (X bridge amide; length, $100 \mathrm{~mm}$; internal diameter, $2.1 \mathrm{~mm}$; particle size, $3.5 \mu \mathrm{m}$; Waters Corp., Milford, MA, USA) at a flow rate of $200 \mu \mathrm{L} / \mathrm{min}$. The injection volume was $3 \mu \mathrm{L}$. The mobile phase consisted of eluent A, Milli-Q water containing $0.2 \%(\mathrm{v} / \mathrm{v})$ formic acid, and eluent $\mathrm{B}$, acetonitrile containing $0.2 \%(\mathrm{v} / \mathrm{v})$ formic acid. The chromatographic run began at 5\% eluent $\mathrm{A}$ and $95 \%$ eluent $\mathrm{B}$, followed by a 5-min linear gradient to $35 \%$ eluent $\mathrm{A}$, a 4-min linear gradient to $75 \%$ eluent $\mathrm{A}$, a 2-min hold, and a 2-min linear gradient to $5 \%$ eluent $\mathrm{A}$. The mobile phase remained at 5\% eluent $\mathrm{A}$ for $27 \mathrm{~min}$ until the end of the analytical run. The MS/MS analysis was performed in positive ion mode. The detected fragment ion occurred at $m / z 166.1 \rightarrow 120.2$.

\section{Quantification of chloramines and free chlorine}

Monochloramine, dichloramine, and free chlorine were quantified by using the $N, N$-diethyl- $p$ phenylenediamine (DPD) colorimetric method (American Public Health Association/American Water Works Association/Water Environment Federation 2005). Trichloramine was quantified by means of headspace-gas chromatography/mass spectrometry (GC/MS) analysis according to Kosaka et al. (2010) by using a GC/MS (GC, 7890A; MS, 5975C; Agilent Technologies, Inc., Palo Alto, CA, USA) coupled with a capillary column (HP-1 MS; length, $30 \mathrm{~m}$; internal diameter, $0.25 \mathrm{~mm}$; thickness, $0.25 \mu \mathrm{m}$; Agilent Technologies). The injection volume was $1.5 \mathrm{~mL}$. The detected fragment ions of trichloramine and 1,1,2-trichloroethane- $\mathrm{d}_{3}$ (internal standard) occurred at $\mathrm{m} / \mathrm{z} 84$ and 100, respectively.

\section{Recovery of NCPAAI, PAA, and PAN both in the LLE and in the SPME}

Fifty milliliters of the high-NCPAAI solution was supplemented with $5 \mathrm{~mL}$ of chloroform and then intensely shaken for 5, 30, 60, and $90 \mathrm{~min}$ by using a mechanical shaker. The chloroform phase was dehydrated with anhydrous sodium sulfate. The resulting chloroform phase was mixed with phosphate buffer and acetone at a ratio of 1:1:10, whereas the high-NCPAAI solution was mixed with fresh chloroform and acetone at a ratio of 1:1:10. The mixtures were directly subjected to LC/MS 
analysis (a hybrid quadrupole-orbitrap mass spectrometer (Q Exactive, Thermo Fisher Scientific Inc., Waltham, MA, USA) coupled with liquid chromatography (UltiMate 3000 LC systems, Thermo Fischer Scientific), and the peak areas of NCPAAI, PAA, and PAN were recorded. TP recoveries were determined by dividing the peak area of a TP in the solution prepared with the chloroform extract by that in the solution prepared directly with the high-NCPAAI solution.

(a) PAN

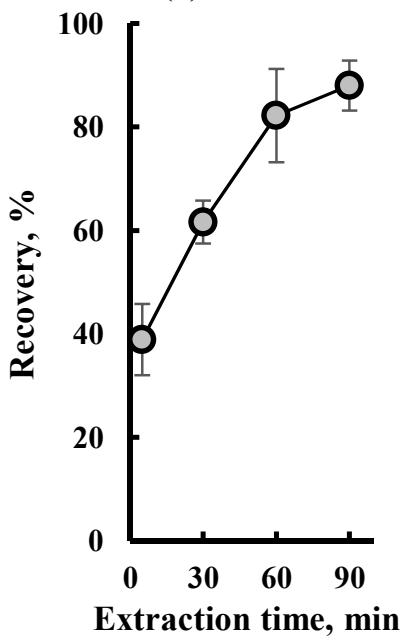

(b) PAA

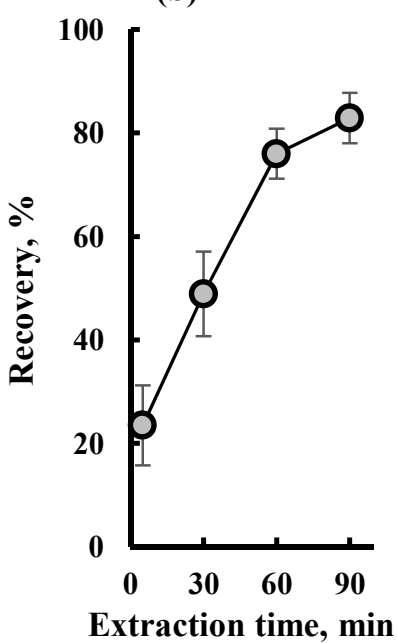

(c) NCPAAI

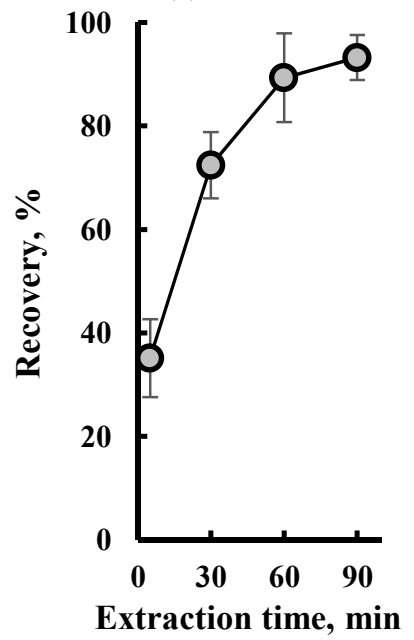

Fig. S1 Changes in the recovery of target compounds with extraction time. PAN, phenylacetonitrile; PAA, phenylacetaldehyde; NCPAAI, $N$-chlorophenylacetaldimine. Error bars indicate the standard deviations of 5 runs.

As shown in Fig. S1, the recoveries of all of the compounds increased with extraction time. At 90 min of extraction time, the recoveries of PAN, PAA, and NCPAAI were determined to be 88,83 , and 93\%, respectively. The recoveries in the SPME were assumed to be the same as each other for all TPs.

\section{${ }^{15} \mathrm{~N}$ mass balance experiments}

${ }^{15} \mathrm{~N}$-labelled phenylalanine was dissolved in phosphate buffer $(0.1 \mathrm{mM}, \mathrm{pH} 7.0)$ at a final concentration of $1 \mu \mathrm{M}$. The phenylalanine-containing solution was supplemented with sodium hypochlorite so as to achieve a residual chlorine concentration at $1 \mathrm{mg}-\mathrm{Cl}_{2} / \mathrm{L}$ after $24 \mathrm{~h}$ of contact time. The solution was stirred with a magnetic stirrer for $30 \mathrm{~min}$ and then left at rest without headspace for $24 \mathrm{~h}$ at $20^{\circ} \mathrm{C}$ in the dark.

The laboratory-prepared, ${ }^{15} \mathrm{~N}$-labelled phenylalanine solution, which contained ${ }^{15} \mathrm{~N}_{2}$ as well as ${ }^{15} \mathrm{~N}$ labelled TPs, was transferred to the lower chamber of a glass vessel with separable upper and lower chambers. The lower chamber was tightly sealed with a separator made of aluminum foil (Fig. S2 A). The gas in the upper chamber of the vessel was replaced with Ar gas. To purge the ${ }^{15} \mathrm{~N}_{2}$ dissolved in the solution into the gas phase, the solution was intensely mixed for $20 \mathrm{~min}$ with the gas phase after the separator was removed and was then kept in a stationary mode for $30 \mathrm{~min}$ (Fig. S2B). An aliquot of the gas was withdrawn from the upper chamber to measure the ${ }^{15} \mathrm{~N}_{2}$ concentration (Fig. S2 C). The same steps corresponding to Figure S2 A-C were repeated (Fig. S2 D-F) to purge the ${ }^{15} \mathrm{~N}_{2}$ completely 
from the solution. An aliquot of the solution was then withdrawn from the lower chamber (Fig. S2 G) to measure the initial concentrations of the TPs, and then an aliquot of phosphate buffer with the same volume as the aliquot that had been withdrawn was added to the solution to ensure that there was no headspace between the surface of the solution and the separator. The solution was supplemented with $3 \mathrm{mg} / \mathrm{L}$ of SPAC (Fig. S2 H), tightly sealed with the separator, and then mixed for 30 min with a magnetic stirrer (Fig. S2 I). During mixing, the gas in the upper chamber was replaced with Ar gas (Fig. S2 J). An aliquot of the gas in the chamber was withdrawn to measure the initial concentration of ${ }^{15} \mathrm{~N}_{2}$. After 30 min of mixing, the separator was removed. The liquid phase was intensely mixed with the gas phase for $20 \mathrm{~min}$ and then left in a stationary mode for 30 min to allow any ${ }^{15} \mathrm{~N}_{2}$ gas generated by the reaction between the TPs and the SPAC to transfer to the gas phase from the solution (Fig. S2 K). An aliquot of the gas phase was withdrawn from the upper chamber to measure the ${ }^{15} \mathrm{~N}_{2}$ concentrations (Fig. S2 L). To measure the TP concentrations, an aliquot of the solution was withdrawn (Fig. S2 M) and then filtered through a membrane filter $(\phi=0.2 \mu \mathrm{m}$, PTFE, Toyo Roshi Kaisha, Ltd., Tokyo, Japan) to remove any SPAC particles. This experiment was conducted at $20{ }^{\circ} \mathrm{C}$.

The concentration of ${ }^{15} \mathrm{~N}_{2}$ in the gas phase was measured with a GC/MS (GC, 7890A; MS, 5975C; Agilent Technologies) equipped with a CP-Molsieve $5 \AA$ capillary column $(50 \mathrm{~m} \times 320 \mu \mathrm{m}, 30 \mu \mathrm{m}$; Agilent Technologies). The temperatures of the ion source, injector, and transfer line were maintained at 230,200 , and $270{ }^{\circ} \mathrm{C}$, respectively. The GC/MS was operated in the SIM mode with ${ }^{38} \mathrm{Ar}$ as an internal standard; the detected fragment ions of ${ }^{15} \mathrm{~N}_{2}$ and ${ }^{38} \mathrm{Ar}$ occurred at $m / z 30$ and 38, respectively.

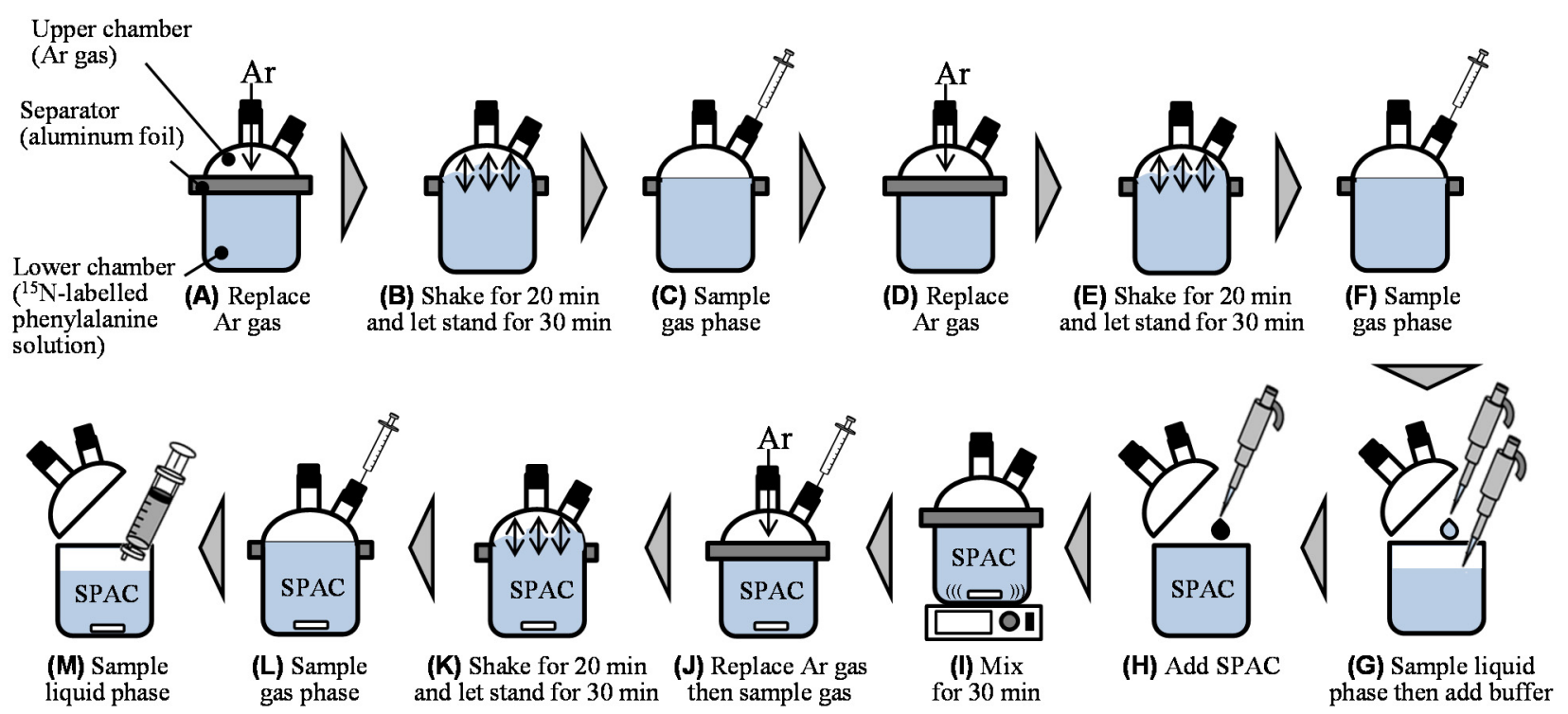

Fig. S2 Protocol for the ${ }^{15} \mathrm{~N}$ mass balance experiments. 


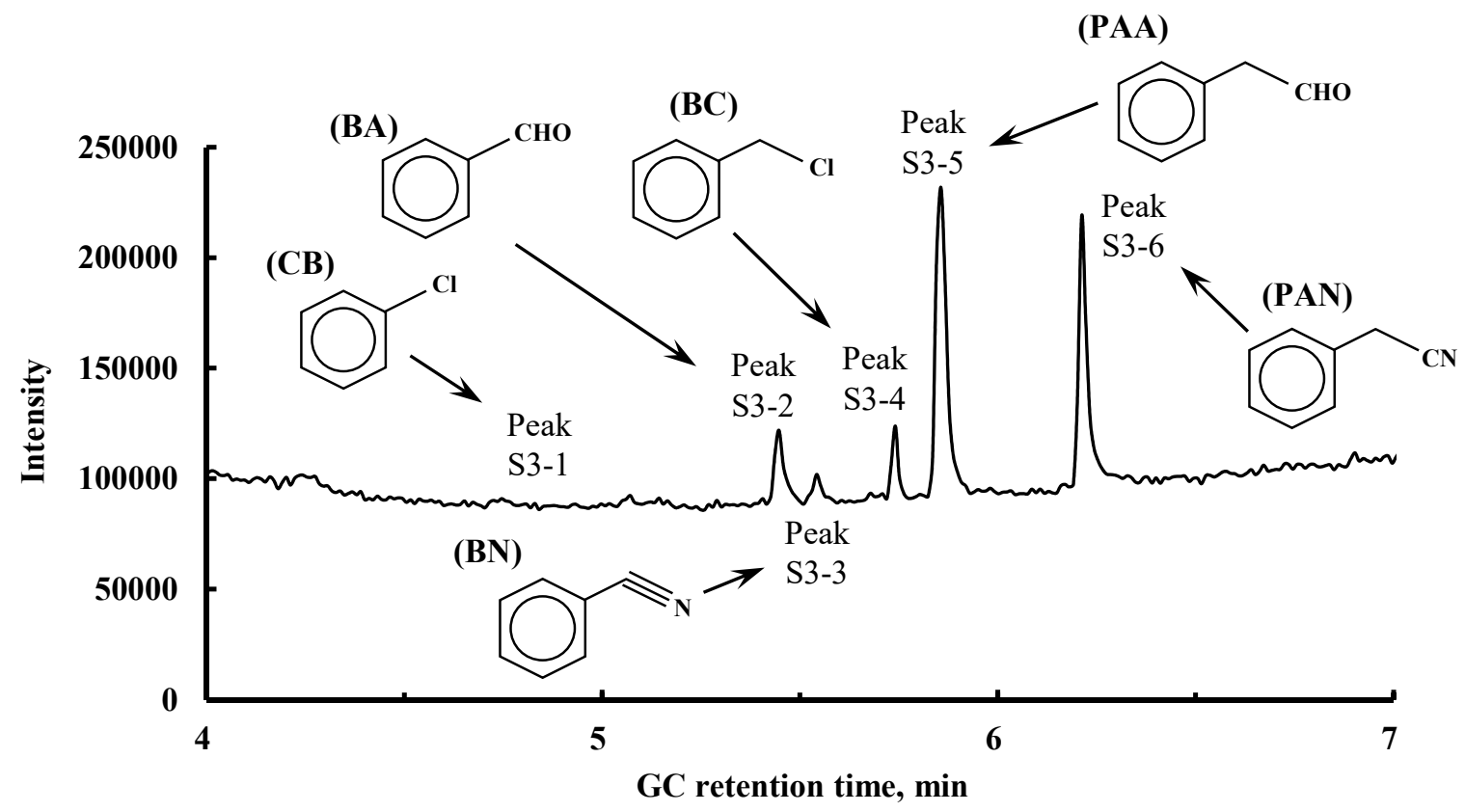

Fig. S3 Purge-and-trap GC/MS TIC chart of the chlorinated phenylalanine-containing solution. CB, chlorobenzene; BA, benzaldehyde; BN, benzonitrile; BC, benzyl chloride; PAA, phenylacetaldehyde; PAN, phenylacetonitrile.

\section{Statistical analysis on the recovery of benzene ring}

As shown in Fig. S4(a), the concentrations of PAA and PAN were measured at $0.57 \pm 0.088$ and $0.31 \pm 0.068 \mu \mathrm{M}$, respectively. Based on the measurements, the concentration of benzene ring detected as PAA or PAN after chlorination ("PAA + PAN" in Fig. S4(b)) was calculated at $0.88 \pm 0.11 \mu \mathrm{M}$. Assuming that the standard deviation of the concentration of benzene ring of the quantifiable TPs (i.e., PAA, PAN, and the minor TPs) is the same to that of "PAA + PAN" because the effect of the minor TPs on the standard deviation is expected to be very small due to their very small concentrations, the concentration of benzene ring of the quantifiable TPs was determined at $0.93 \pm 0.11$ $\mu \mathrm{M}$ ("PAA + PAN + minor TPs" in Fig. S4(b)). Of course, statistical analysis on the difference in the concentration of benzene ring before and after chlorination should not be carried out directly, because of the fact that the concentration of phenylalanine before chlorination was measured only once adding to the above-mentioned assertive assumption (i.e., the standard deviations were the same between " PAA + PAN" and "PAA + PAN + minor TPs"). However, judging from Fig. S4(b), detailed discussion on the difference was likely to be avoided: i.e., the difference (7\%) may be within the experimental error. 
(a)

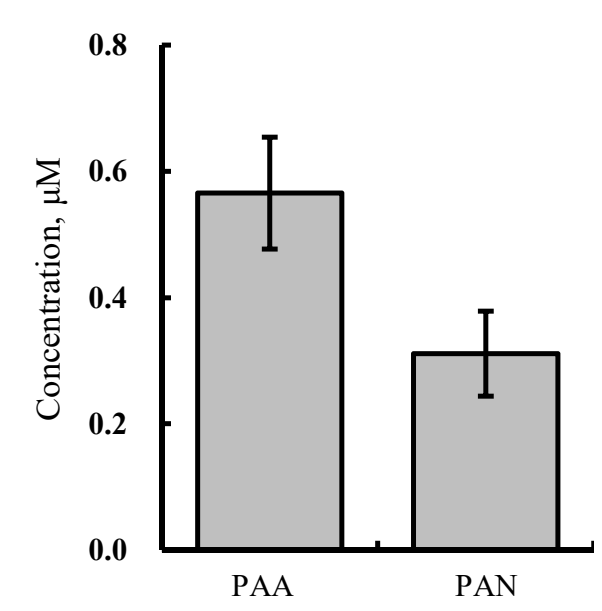

(b)

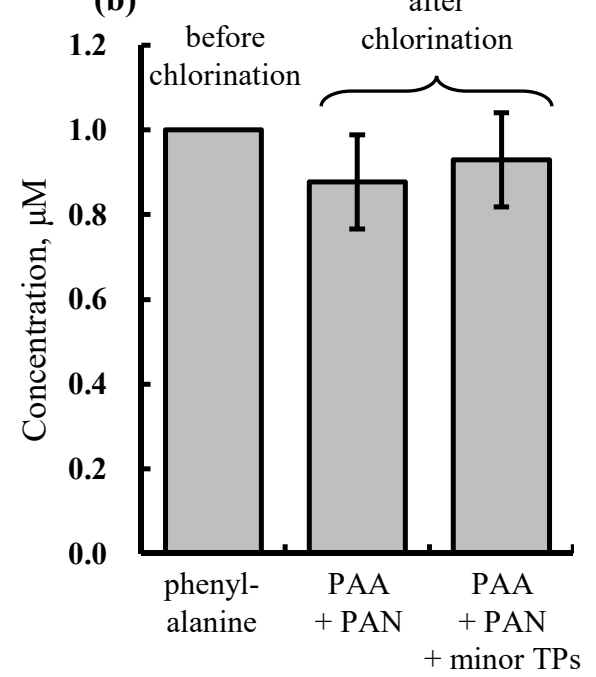

Fig. S4 Error analyses in P\&T-GC/MS. (a) Concentrations of PAA and PAN detected in the chlorinated phenylalanine-solution. (b) Mass balance of benzene ring before and after chlorination. Error bars represent standard deviations of 17 measurements. The standard deviation of "PAA + PAN + minor TPs" was assumed to be the same as that of "PAA + PAN".

Comparison of off-flavor intensity as determined by using the conventional flask test between the chlorinated phenylalaninecontaining solution and the manufactured solutions containing TPS

To investigate possible synergistic effects among the TPs, a sensory evaluation using the conventional flask test was performed on contrived solutions that contained PAN + PAA + free chlorine, and PAN + PAA + free chlorine + trichloramine at the same concentrations as those found in the chlorinated phenylalaninecontaining solution. As shown in Fig. S5, the observed off-flavor intensities of the contrived solutions were almost the same as those calculated from the concentrations and odor thresholds of the TPs contained in the solutions: no synergistic effect was observed among the TPs tested here in terms of inducing off-flavor.

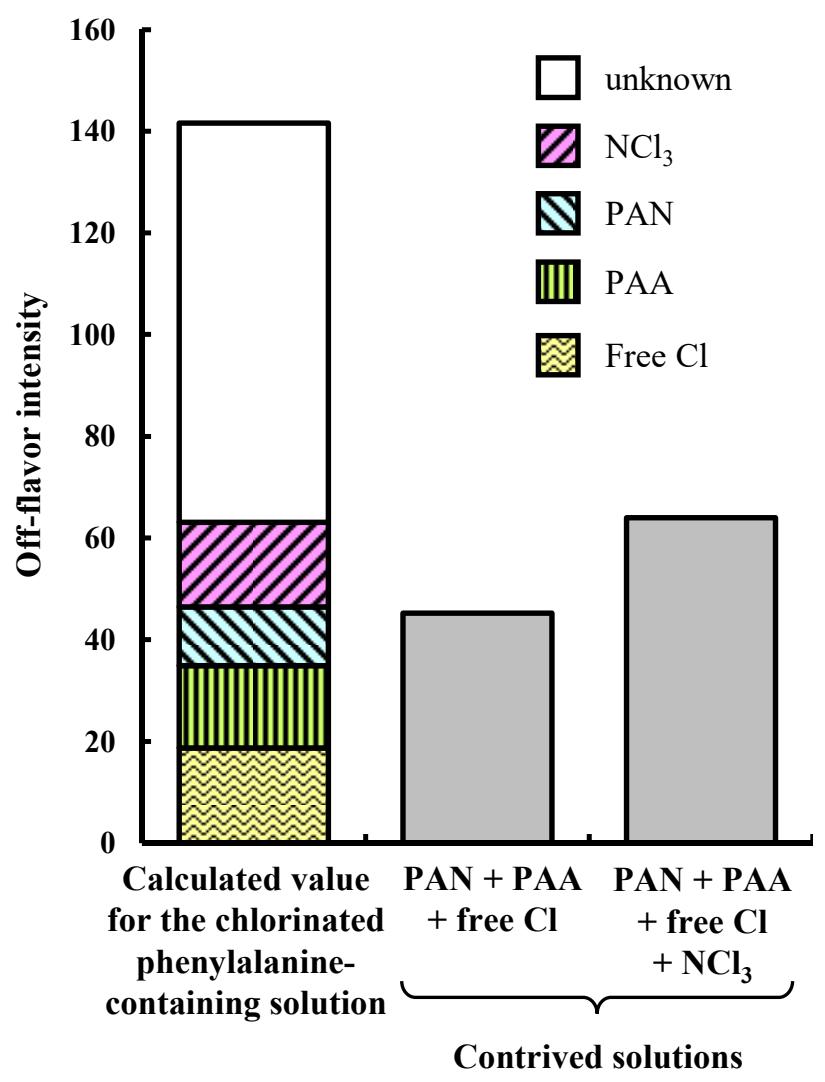

Fig. S5 Off-flavor intensities of the contrived solutions containing $\mathrm{PAN}+\mathrm{PAA}+$ free $\mathrm{Cl}$, and $\mathrm{PAN}+\mathrm{PAA}+$ free $\mathrm{Cl}+\mathrm{NCl}_{3}$ at the same concentrations as those in the chlorinated phenylalanine-containing solution. PAN, phenylacetonitrile; PAA, phenylacetaldehyde; $\mathrm{NCl}_{3}$, trichloramine; free $\mathrm{Cl}$, free chlorine. 
Mass spectrum of Peak 6-3 detected in the chlorinated phenylalanine-containing solution

Fig. S6 shows the mass spectrum of Peak 6-3 depicted in Fig. 6. Conyers et al. (1993) previously reported the mass spectrum of NCPAAI. The mass spectrum obtained in the present study was slightly different from that reported by Conyers et al. (1993), but the fragment ions that were detected at high intensities in the present study corresponded to those reported by Conyers et al. (1993): $\mathrm{m} / \mathrm{z} 51,65$, 91, 118, and 153. Moreover, the same peak (retention time and mass spectrum) was obtained in the high-NCPAAI solution at a much higher intensity. These findings strongly suggested that the TP detected as Peak 6-3 was NCPAAI.

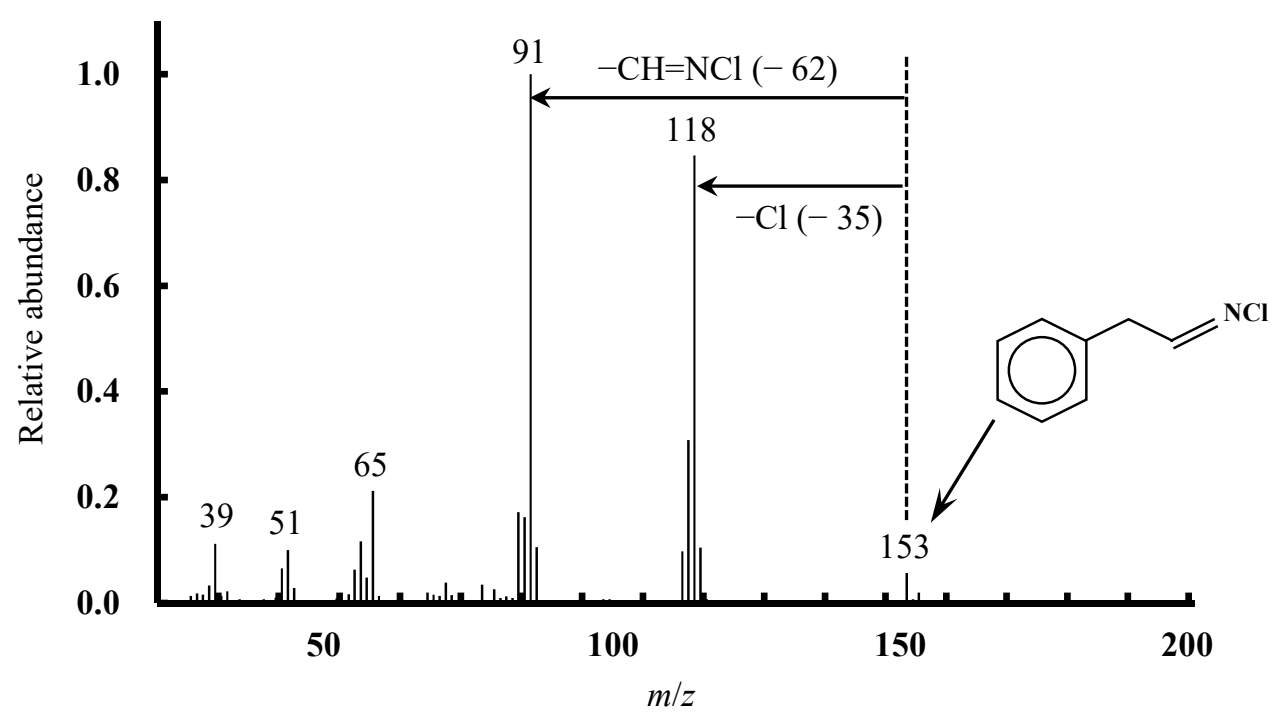

Fig. S6 Mass spectrum of Peak 6-3 in Fig. 6.

\section{Estimation of the chemical structure of the TP detected as Peak 7-4}

The TP detected as Peak 7-4 was suspected to contribute to the off-flavor. From the mass spectrum of Peak 7-4 (Fig. S7(a)), the monoisotopic nominal molecular weight of the TP was found to be 154 . Because the monoisotopic nominal molecular weight of the TP was even, the TP had no nitrogen atom according to the nitrogen rule. The fact that the intensity of $\mathrm{m} / \mathrm{z} 156$ was approximately onethird that of $m / z 154$ strongly suggested that the TP had one chlorine atom in its structure. From these data, combined with the chemical structure of the parent compound phenylalanine, four types of possible chemical structure could be proposed for the TP detected as Peak 7-4 (Table S1).

Although the chemical standards for 2-(2-chlorophenyl)acetaldehyde and 2-chloro-2phenylacetaldehyde were not commercially available, those for 2-(3-chlorophenyl)acetaldehyde and 2-(4-chlorophenyl)acetaldehyde were. The latter two compounds were purchased, individually dissolved in Milli-Q water, and subjected to the SPME followed by GC-MS-O analysis. Similar fragment ions were observed in the mass spectra of these two compounds and Peak 7-4, but their patterns were somewhat different from each other (Fig. S7). Moreover, the GC retention times of these two compounds (6.7-6.8 min) were far from that of Peak 7-4 (6.2 min). These observations 
clearly indicated that the TP detected as Peak 7-4 was neither 2-(3-chlorophenyl)acetaldehyde nor 2(4-chlorophenyl)acetaldehyde.
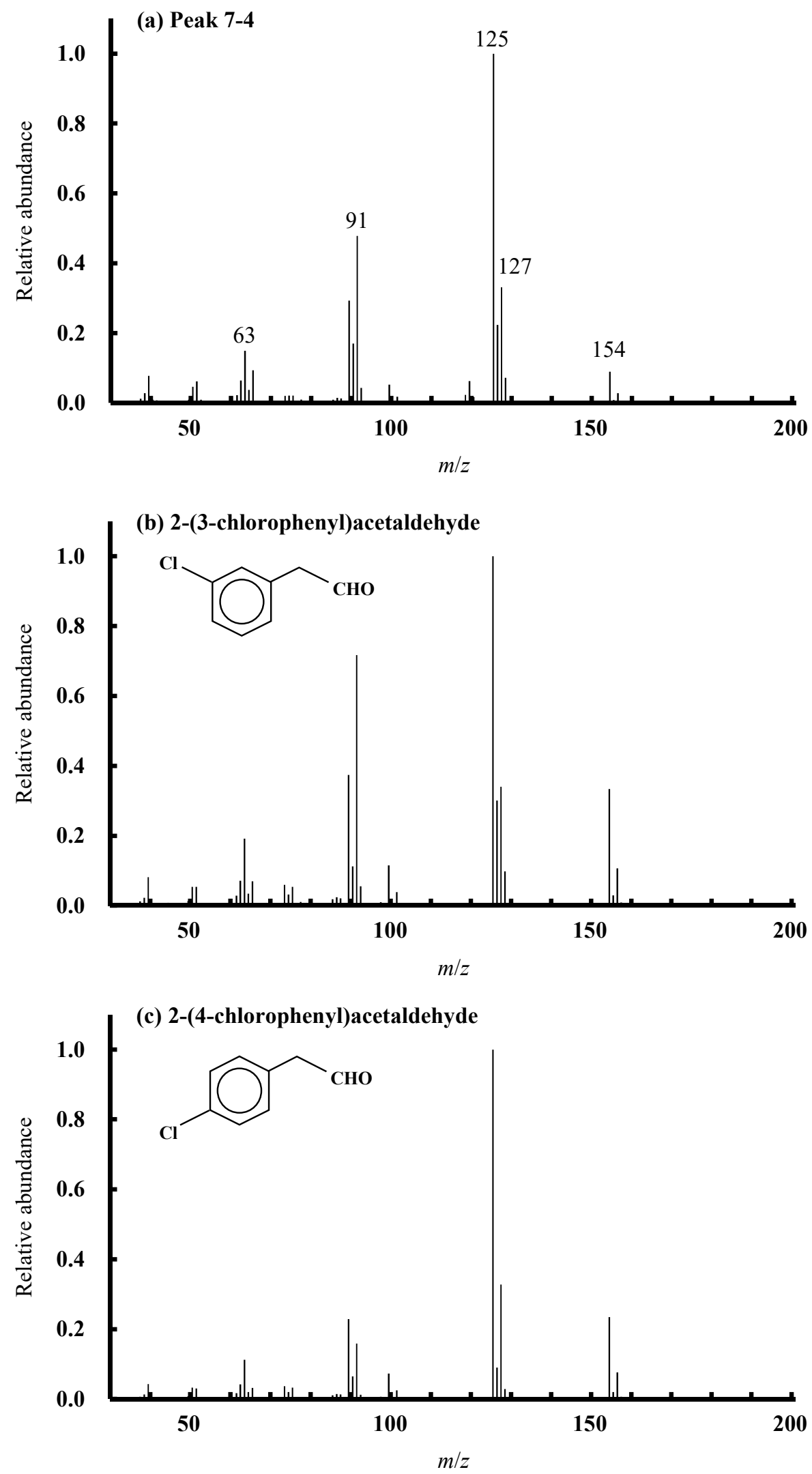

Fig. S7 Comparison of the mass spectra of (a) Peak 7-4 $(\mathrm{RT}=6.2 \mathrm{~min})$, (b) the chemical standard for 2-(3-chlorophenyl)acetaldehyde $(\mathrm{RT}=6.7 \mathrm{~min})$, and $(\mathrm{c})$ the chemical standard for 2-(4-chlorophenyl)acetaldehyde $(\mathrm{RT}=6.8 \mathrm{~min})$. 
According to the information provided by the supplier and the published literature (Flego and Zannoni, 2011), the GC column used in the present study (HP-1ms) periodically separates compounds according to their boiling point: compounds elute in the order of their increasing boiling points when the compounds have similar structures, functional groups, or are part of an homologous series. Because 2-(2-chlorophenyl)acetaldehyde had the same boiling point as 2-(3chlorophenyl)acetaldehyde and 2-(4-chlorophenyl)acetaldehyde (Table S1), the GC retention times of these three compounds were expected to be similar. In contrast, the boiling point of 2-chloro-2phenylacetaldehyde was lower than that of 2-(3-chlorophenyl)acetaldehyde and that of 2-(4chlorophenyl)acetaldehyde. This suggests that the GC retention time of 2-chloro-2phenylacetaldehyde was most likely shorter than those of 2-(3-chlorophenyl)acetaldehyde and 2-(4chlorophenyl)acetaldehyde. The fact that the GC retention time of Peak 7-4 was shorter than those of 2-(3-chlorophenyl)acetaldehyde and 2-(4-chlorophenyl)acetaldehyde suggests that the TP represented by Peak 7-4 was not 2-(2-chlorophenyl)acetaldehyde but 2-chloro-2-phenylacetaldehyde.

Table S1 Possible chemical structures of the TP detected as Peak 7-4. Boiling points were calculated by using ACD/Labs Percepta Predictor and the EPISuite on the ChemSpider website (http://www.chemspider.com/).

BCD/Labs
Boiling point, ${ }^{\circ} \mathrm{C}$
EPISuite

(a) 2-(2-chlorophenyl)acetaldehyde<smiles>O=CCc1cccc(Cl)c1</smiles>

(b) 2-(3-chlorophenyl)acetaldehyde<smiles>O=CCc1ccc(Cl)cc1</smiles>

(c) 2-(4-chlorophenyl)acetaldehyde<smiles>O=CC(Cl)c1ccccc1</smiles>

(d) 2-chloro-2-phenylacetaldehyde 
Mass balance of ${ }^{15} \mathrm{~N}$ before and after SPAC treatment of chlorinated phenylalanine-containing solution

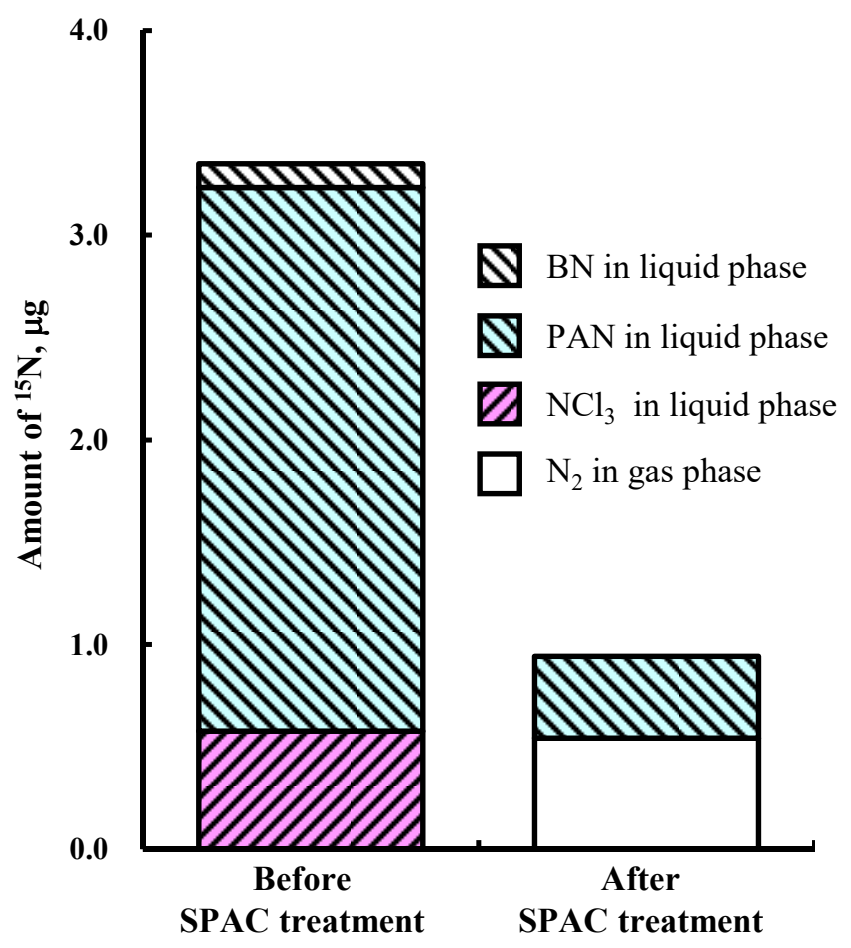

Fig. S8 Comparison of the amount of ${ }^{15} \mathrm{~N}$ in quantifiable TPs before and after SPAC treatment of chlorinated phenylalanine-containing solution. $\mathrm{BN}$, benzonitrile; PAN, phenylacetonitrile; $\mathrm{NCl}_{3}$, trichloramine.

\section{References}

Conyers, B. and Scully, F.E. (1993) N-chloroaldimines. 3. Chlorination of phenylalanine in model solutions and in a wastewater. Environmental Science \& Technology 27, 261-266.

Flego, C. and Zannoni, C. (2011) N-containing species in crude oil fractions: An identification and quantification method by comprehensive two-dimensional gas chromatography coupled with quadrupole mass spectrometry. Fuel 90, 2863-2869. 